\title{
Arbitrary axisymmetric steady streaming: Flow, force and propulsion
}

\author{
Tamsin A. Spelman • Eric Lauga
}

14 th September 2016

\begin{abstract}
A well-developed method to induce mixing on microscopic scales is to exploit flows generated by steady streaming. Steady streaming is a classical fluid dynamics phenomenon whereby a time-periodic forcing in the bulk or along a boundary is enhanced by inertia to induce a non-zero net flow. Building on classical work for simple geometrical forcing and motivated by the complex shape oscillations of elastic capsules and bubbles, we develop the mathematical framework to quantify the steady streaming of a spherical body with arbitrary axisymmetric time-periodic boundary conditions. We compute the flow asymptotically for small-amplitude oscillations of the boundary in the limit where the viscous penetration length scale is much smaller than the body. In that case, the flow has a boundary layer structure and the fluid motion is solved by asymptotic matching. Our results, presented in the case of no-slip boundary conditions and extended to include the motion of vibrating free surfaces, recovers classical work as particular cases. We illustrate the flow structure given by our solution and propose one application of our results for small-scale force-generation and synthetic locomotion.
\end{abstract}

\section{Introduction}

Flow mixing and transport on small scales have been widely studied [1]. These processes can be non-trivial as small-scale flows often occur at low Reynolds number and as such are laminar and time-reversible. Achieving precise flow and mixing control is however critical experimentally in a range of medical and chemical applications [2]. Applying pressure differences or inducing electrokinetic flows are the two most common methods used to drive flows on small scales [1] and mixing has often been achieved by encouraging chaotic particle paths e.g. by designing a device with complex geometries [3,2].

Department of Applied Mathematics and Theoretical Physics, University of Cambridge, Wilberforce Road, Cambridge, CB3 0WA, UK. E-mail: e.lauga@damtp.cam.ac.uk 
As an alternative, one can look at the biological world for inspiration in design since mixing and transport occurs on cellular length scales [4]. Individual microorganisms are known to generate a net dipolar flow in the surrounding fluid when they self-propel $[5,6]$. A population of swimmers generates larger more varied flows, which can aid mixing, through a variety of processes including bioconvection [7] and purely collective effects [8,9]. More subtly their presence may affect the diffusive transport of passive tracers which develops non-Gaussian tails in the presence of biological activity $[10,11,12]$.

From a modelling standpoint, analytic studies of flows induced by swimmers is older than experimental observations due to previous limitations of imaging techniques. Many approximately spherical microorganisms (e.g. the multicellular alga Volvox or the protozoon Opalina) move by waving slender appendages called cilia. A standard modelling approach consists in considering the dynamics of the enclosing envelope of the cilia, thus reducing the problem to that of a spherical body inducing a surface wave of deformation [13,14]. The first of such analytic models was proposed by Lighthill [15] and later corrected by Blake [13], who calculated the net flow generated by small-amplitude axisymmetric oscillations of a spherical surface in a Stokes flow. This model is now refereed to as the "squirmer" model. More recent studies have extended this model to include non-axisymmetric motion [16], the presence of nearby boundaries $[17,18]$ and large-amplitude oscillations [19].

Recently the importance of inertia to the swimming of microorganisms was recognised. Unsteady inertial effects are particularly influential over short time scales [20] but not on longer timescales [21]. A theoretical extension of Lighthill's original model to include unsteady inertia was carried out by Rao [22]. Convective inertia, in contrast, is important for larger microorganisms, typically of radius close to one millimetre. Using a squirmer model with tangential forcing on the fluid only, two recent studies considered how convective inertia impacted the swimming kinematics and efficiency, emphasizing in particular the relationship between the direction of the far-field flow and the inertial effects $[23,24]$

In the lab, a variety of synthetic drivers and mixers at the micron-scale have been designed and experimentally tested [25,26]. The simplest devices are time-periodic and exploit the inertia in the fluid to generate net motion. This motion is the classical phenomenon of steady (or acoustic) streaming, whereby a time-periodic forcing is nonlinearly rectified by inertia to induce a non-zero net flow [27]. One method to obtain this time periodic forcing is to use microbubbles whose surfaces oscillate when forced with ultrasound, allowing the transformation of a macroscopic piezoelectric forcing into oscillations at the micrometer scale, with a range of potentially important biomedical applications [28]. Studies of the streaming generated by oscillating microbubbles started with Elder's experiments in 1958 [29]. Microbubbles are able to transport particles either in their streaming flow when the microbubble is held stationary $[30,31]$ or where the microbubble itself carries the particle [28, 32]. Interactions between multiple oscillating bubbles may be used to increase mixing flows $[33,34,35]$. 
Theoretical studies of the steady streaming flows have focused on shape oscillations in simple geometries, including a translating sphere [36], a translating bubble [37,38], a bubble both translating and pulsating [38], and more recently a bubble both pulsating and oscillating with one higher-order Legendre mode [39]. For free microbubbles, the external acoustic energy is focused into the first few surface modes of oscillation hence these classical studies are sufficient to model streaming. However as setups become more complicated, for example in the case of solid capsules partially enclosing three-dimensional bubbles $[32,40,41]$, it is important to be able to model the complex shape dynamics and accurately compute the resulting streaming flows and forces.

In this paper, we develop the mathematical framework to quantify the steady streaming of a spherical body with arbitrary axisymmetric time-periodic boundary conditions (see Figure 1 for setup). We compute the flow asymptotically under two assumption: (1) the amplitude of surface oscillations are small relative to the size of the body (ratio of amplitude $\epsilon \ll 1$ ); and (2) the acoustic frequency is large such that the viscous penetration length scale is small compared to the body size (ratio penetration length to body size $\delta \ll 1$ ). Mathematically, for a fixed value of $\delta$, we solve the flow in powers of $\epsilon$, and thus focus implicitly on the limit $\epsilon \ll \delta \ll 1$. This limit is the relevant one for micron-size bubbles forced by ultrasound (frequencies in the hundreds of $\mathrm{kHz}$ range) and millimetre-sized organisms. Similarly to classical work, the flow is shown to have a boundary layer structure and the problem is solved by asymptotic matching. Our results, which assume the body is fixed in space, are presented in the case of no-slip boundary conditions and extended to include the motion of vibrating free surfaces, also recover classical work as particular cases. We then illustrate the flow structure given by our solution and propose one application of our results, discussing the adaptation for a force-free body, on small-scale force-generation and synthetic locomotion.

Our paper is organised as follows. In $\S 2$ we set up the problem of the fluid flow generated by arbitrary surface motion of a no-slip spherical body. In $\S 3$, we derive the first-order solution. The second-order Eulerian steady streaming is derived in $\S 4$ which is extended to give the Lagrangian steady streaming in $§ 5$. The special case of a squirming microorganism is then discussed in $\S 6$. This no-slip general model can be extended to incorporate other surface motion such as a no tangential stress boundary as shown in $\S 7$. The special case of a bubble is then considered in $\S 8$. In $\S 9$ our general solution is validated against classical results for spheres and bubbles. In $\S 10$, we illustrate examples of streaming flows. In all previous sections, the body was assumed to be held stationary at the origin. In $\S 11$ the time-averaged force induced by the flow on the fixed body is calculated, along with the translational velocity of the spherical body if it was instead free to move. 

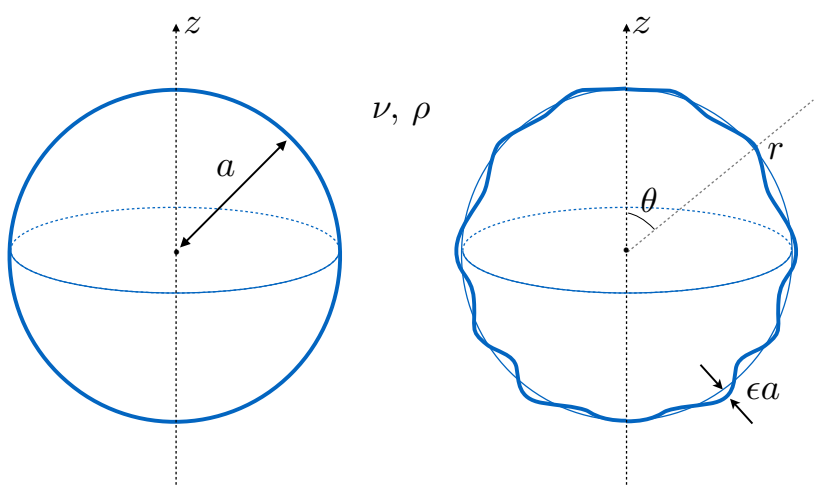

Fig. 1: A sphere of rest radius a (left) undergoes arbitrary axisymmetric vibrations of amplitude $\epsilon a$, with $\epsilon \ll 1$ (right). Spherical coordinates are used with radial distance $r$ and polar angle $\theta$. The surrounding fluid is Newtonian with kinematic viscosity $\nu$ and density $\rho$.

\section{Axisymmetric steady streaming: Setup}

In this first section we present the general setup for our calculation. The body is taken to be spherical with an imposed axisymmetric, radial and tangential time-periodic deformation of its surface. In the following sections we will use asymptotic matching to first characterise the flow in the case of no-slip between the fluid and the surface, and then we generalise to allow the formulation to be adapted to other boundary conditions, in particular no tangential stress for a clean bubble.

\subsection{Statement of the mathematical problem}

The sphere has mean radius $a$ and is contained within an unbounded Newtonian fluid of constant kinematic viscosity $\nu$ and uniform density $\rho$ (Figure 1 ). Working in a spherical coordinate system centred on the sphere, with radial distance $r$ and polar angle $\theta$, the axis $\theta=0$ is the axis of rotational symmetry. The surface of the body is assumed to oscillate at angular frequency $\omega$ with small amplitude $\epsilon a$, where $\epsilon \ll 1$ is formally specified below. Define $\mu \equiv \cos \theta$, then since the flow is axisymmetric a streamfunction $\psi$ can be introduced to give radial $u_{r}$ and angular $u_{\theta}$ velocities as

$$
\begin{aligned}
& u_{r}=-\frac{1}{r^{2}} \frac{\partial \psi}{\partial \mu} \\
& u_{\theta}=\frac{-1}{r\left(1-\mu^{2}\right)^{\frac{1}{2}}} \frac{\partial \psi}{\partial r}
\end{aligned}
$$


The governing equation is then given by the vorticity equation [42]

$$
\frac{\partial\left(D^{2} \psi\right)}{\partial t}+\frac{1}{r^{2}}\left[\frac{\partial\left(\psi, D^{2} \psi\right)}{\partial(r, \mu)}+2 D^{2} \psi L \psi\right]=\nu D^{2}\left(D^{2} \psi\right)
$$

where we have defined the operators

$$
\begin{aligned}
D^{2} & \equiv \frac{\partial^{2}}{\partial r^{2}}+\frac{\left(1-\mu^{2}\right)}{r^{2}} \frac{\partial^{2}}{\partial \mu^{2}}, \\
L & \equiv \frac{\mu}{\left(1-\mu^{2}\right)} \frac{\partial}{\partial r}+\frac{1}{r} \frac{\partial}{\partial \mu} .
\end{aligned}
$$

In order to non-dimensionalise the problem, we take the relevant time scale to be $\omega^{-1}$ and the relevant length scale to be $a$, so that the sphere now has a rest radius of 1 . The streamfunction thus has dimensions $a^{3} \omega$ and the vorticity equation becomes

$$
\frac{\partial\left(D^{2} \psi\right)}{\partial t}+\frac{1}{r^{2}}\left[\frac{\partial\left(\psi, D^{2} \psi\right)}{\partial(r, \mu)}+2 D^{2} \psi L \psi\right]=\left(\frac{\nu}{\omega a^{2}}\right) D^{2}\left(D^{2} \psi\right)
$$

Equation (6) introduces a non-dimensional quantity: the ratio of the viscous penetration length scale, $\sim(\nu / \omega)^{1 / 2}$, to the radius of the body, $a$. Specifically, we define a dimensionless number $\delta$ as

$$
\delta \equiv \frac{(2 \nu / \omega)^{\frac{1}{2}}}{a}
$$

thus reducing the governing equation to

$$
\frac{\partial\left(D^{2} \psi\right)}{\partial t}+\frac{1}{r^{2}}\left[\frac{\partial\left(\psi, D^{2} \psi\right)}{\partial(r, \mu)}+2 D^{2} \psi L \psi\right]=\left(\frac{\delta^{2}}{2}\right) D^{2}\left(D^{2} \psi\right)
$$

The second dimensionless quantity in this problem is the ratio $\epsilon$ between the amplitude of oscillation and the body radius. To use notations similar to those in classical steady streaming calculations, we use $U$ to denote the maximum velocity at the surface of the oscillating body, such that $\epsilon$ can be defined as

$$
\epsilon=\frac{U}{a \omega} \text {. }
$$

We will look to solve this problem as a regular expansion in $\epsilon$ for small values of $\delta$, and then take a regular expansion in $\delta$. We will thus assume the asymptotic limit $\epsilon \ll \delta \ll 1$. Importantly, this assumption is sufficient for our asymptotic solution to be valid. As explained below, we will solve this problem using asymptotic matching between an inner solution (boundary layer of size $\delta$ ) and an outer solution. For the inner asymptotic solution, $\psi^{i}$, where we require at least one more term than in the outer solution we will have an expansion of the form

$$
\psi^{i}=\epsilon\left(\psi_{1}^{i(0)}+\delta \psi_{1}^{i(1)}+\ldots+\delta^{n} \psi_{1}^{i(n)}\right)+\epsilon^{2}\left(\psi_{2}^{i(0)}+\delta \psi_{2}^{i(1)}\right)+O\left(\epsilon \delta^{n+1}, \epsilon^{2} \delta^{2}, \epsilon^{3}\right),
$$


with $n \geq 2$. The solution in (10) is a valid approximation provided the errors are smaller than the order of our solution $O\left(\epsilon^{2} \delta\right)$. So we require $\delta \ll 1, \epsilon \ll \delta$ and $\delta^{n} \ll \epsilon$. But $n$ can be chosen to be as large as required, thus reducing down to the condition $\epsilon \ll \delta \ll 1$ only. We note that we can easily obtain the $O(\epsilon)$ solution up to order $n$ in $\delta$ since $\epsilon$ is introduced into our equations only through assuming $\psi$ is a power series in $\epsilon$ so mathematically higher orders of $\epsilon$ cannot effect lower orders in $\epsilon$.

Physically, a small value of $\epsilon$ indicates small-amplitude motion while a small value of $\delta$ means that the viscous penetration length is small compared to the rest size of the body. For which practical situations will these limits be relevant? To fix ideas, let us take a value for the relative amplitude of $\epsilon \sim 10^{-2}$. A ciliated microorganism in water $\left(\nu=10^{-6} \mathrm{~m}^{2} / \mathrm{s}\right)$ would have intrinsic frequencies of about $50 \mathrm{~Hz}$, so $\omega \approx 300 \mathrm{rad} / \mathrm{s}$, leading to a penetration length of $(2 \nu / \omega)^{\frac{1}{2}} \approx 80 \mu \mathrm{m}$. In order to satisfy the limit $\epsilon \ll \delta \ll 1$, the organism size would need to be just below $1 \mathrm{~mm}$, which is achieved for the largest ciliated organisms such as Spirostomum which can grow up to $4 \mathrm{~mm}$ in length [43]. For a micro bubble actuated by ultrasound the frequency is about $\omega \sim 10^{6} \mathrm{rad} / \mathrm{s}$, so the penetration length is $(2 \nu / \omega)^{\frac{1}{2}} \approx 1 \mu \mathrm{m}$ and thus the bubble would have to be about $10 \mu \mathrm{m}$ in diameter.

\subsection{Boundary conditions}

We apply, in this first part of the paper, the no-slip boundary condition. Thus, the fluid velocity has to match the velocity of the material points on the surface of the body, which is arbitrary and decomposed along an infinite sum of surface modes. Using a Lagrangian formulation, the motion of the boundary can be described by its radial position, $R$, and angular position measured from the axis of axisymmetry, $\Theta$, which are functions of time $t$ and the rest angular position $\theta$ (through $\mu$ ) as

$$
\begin{aligned}
& R=1-\epsilon \sum_{n=0}^{\infty} V_{n} P_{n}(\mu) e^{i\left(t+\frac{\pi}{2}\right)}+O\left(\epsilon^{2}\right), \\
& \Theta=\theta+\epsilon \sum_{n=1}^{\infty} W_{n}\left(\frac{\int_{\mu}^{1} P_{n}(x) d x}{\left(1-\mu^{2}\right)^{\frac{1}{2}}}\right) e^{i\left(t+\frac{\pi}{2}\right)}+O\left(\epsilon^{2}\right),
\end{aligned}
$$

where $V_{n}$ and $W_{n}$ are arbitrary complex constants determined by the surface motion of the spherical body and $P_{n}(x)$ is the Legendre Polynomial of degree $n$. Throughout the paper, complex notation will be used and it will always be implied that only the real part is taken; when an explicit real part appears we will denote it $\Re$.

The $\mu$-dependence was chosen in order to match the form of the first-order solution, as seen below. Through the many possible choices for constants $V_{n}$ and $W_{n}$, a wide range of boundary motions can be studied. At leading order, $R(\theta)$ is equivalent to the radial position of the surface at an angle $\theta$ from 
the axis of symmetry. As such, $V_{n}$ will be determined by the shape of the surface oscillation. At leading order, $\Theta(\theta)$ captures the tangential motion at an angle $\theta$ from the axis of symmetry, and thus $W_{n}$ will be determined by the appropriate in-surface motion. We note that the use of Legendre polynomials as a basis for $\mu$ was expected due to the problem's axisymmetry and such a basis has appeared in other work based in similar regimes [13]. As the Legendre polynomials form a complete orthogonal basis, they allow for an arbitrary solution to be written in this form.

At $O\left(\epsilon^{2}\right)$ only terms in $R$ and $\Theta$ which time-average to a non-zero value would contribute to the streaming. However such terms would indicate that the body was slowly growing or shrinking over time, and would also stretch or contract tangentially, violating the small-amplitude assumption on long time scales. We therefore do not allow for steady Lagrangian terms at order $O\left(\epsilon^{2}\right)$. The boundary condition can thus be written in a Lagrangian form as

$u_{r}=\frac{\partial R}{\partial t}=\epsilon \sum_{n=0}^{\infty} V_{n} P_{n}(\mu) e^{i t}+O\left(\epsilon^{3}\right)$

$u_{\theta}=R \frac{\partial \Theta}{\partial t}=\Re\left[-\epsilon \sum_{n=1}^{\infty} W_{n}\left(\frac{\int_{\mu}^{1} P_{n}(x) d x}{\left(1-\mu^{2}\right)^{\frac{1}{2}}}\right) e^{i t}\right] \Re\left[1-\epsilon i \sum_{n=0}^{\infty} V_{n} P_{n}(\mu) e^{i t}\right]+O\left(\left(\epsilon^{3} 4\right)\right)$

and both of which have to be evaluated at $(r, \theta)=(R(\mu, t), \Theta(\mu, t))$.

Finally, we require that the flow decay to zero from the body and thus $u_{r, \theta} \rightarrow 0$ as $r \rightarrow \infty$.

We note here that we are assuming the spherical body is fixed in space and as such is not force free. A force-free condition may be incorporated with the same setup in a suitable reference frame, whereby it limits the possible surface shape oscillations through restrictions on the choice of constants $V_{n}$ and $W_{n}$. This is discussed in detail in $§ 11$.

\subsection{Rearranging the surface boundary conditions}

The current Lagrangian form of the body's surface conditions (13)-(14) needs to be transformed into Eulerian boundary conditions of the fluid motion. This is achieved by Taylor expanding them about the average oscillation position $(1, \theta)$ which in spherical coordinates gives

$$
\begin{aligned}
\left.u_{r}\right|_{r=R}=\left.u_{r}\right|_{r=1}+ & \Re[(R-1)] \Re\left[\left.\frac{\partial u_{r}}{\partial r}\right|_{r=1}\right] \\
& +\Re[(\Theta-\theta)] \Re\left[\left.\left(\frac{\partial u_{r}}{\partial \theta_{0}}-u_{\theta}\right)\right|_{r=1}\right]+O\left(\epsilon^{3}\right) .
\end{aligned}
$$

and 


$$
\begin{aligned}
\left.u_{\theta}\right|_{r=R}=\left.u_{\theta}\right|_{r=1}+ & \Re[(R-1)] \Re\left[\left.\frac{\partial u_{\theta}}{\partial r}\right|_{r=1}\right] \\
+ & \Re[(\Theta-\theta)] \Re\left[\left.\left(\frac{\partial u_{\theta}}{\partial \theta_{0}}+u_{r}\right)\right|_{r=1}\right]+O\left(\epsilon^{3}\right) .
\end{aligned}
$$

Looking for the dimensionless velocities and streamfunction as power series in $\epsilon$ as

$$
\begin{aligned}
u_{r} & =\epsilon u_{r}^{(1)}+\epsilon^{2} u_{r}^{(2)}+O\left(\epsilon^{3}\right), \\
u_{\theta} & =\epsilon u_{\theta}^{(1)}+\epsilon^{2} u_{\theta}^{(2)}+O\left(\epsilon^{3}\right), \\
\psi & =\epsilon \psi_{1}+\epsilon^{2} \psi_{2}+O\left(\epsilon^{2}\right),
\end{aligned}
$$

then at leading order (15) and (16) gives

$$
\begin{aligned}
& u_{r}^{(1)}=\sum_{n=0}^{\infty} V_{n} P_{n}(\mu) e^{i t}, \\
& u_{\theta}^{(1)}=-\sum_{n=1}^{\infty} W_{n}\left(\frac{\int_{\mu}^{1} P_{n}(x) d x}{\left(1-\mu^{2}\right)^{\frac{1}{2}}}\right) e^{i t},
\end{aligned}
$$

at $r=1$ and for all values of $\theta$. Similarly from (15) and (16) the $O\left(\epsilon^{2}\right)$ boundary condition can be calculated. However due to the non-linear terms arising from the Taylor expansions, the first-order flow needs to be first evaluated in order to determine the $O\left(\epsilon^{2}\right)$ boundary conditions explicitly. This will be discussed in $§ 4.1$.

\section{First-order Asymptotic solution}

\subsection{General solution}

Based on the oscillatory nature of the boundary condition at first order, (20)(21), we look for a solution $\psi_{1} \propto e^{i t}$. At leading order the governing equation reduces to

$$
\left(\frac{\partial}{\partial t}-\frac{\delta^{2}}{2} D^{2}\right)\left(D^{2} \psi_{1}\right)=0
$$

This is easily solved using separation of variables $D^{2} \psi_{1}=f(r) g(\mu) e^{i t}$ to find $D^{2} \psi_{1}$ as

$$
D^{2} \psi_{1}=e^{i t}\left[\sum_{n=1}^{\infty}\left(\int_{\mu}^{1} P_{n}(x) d x\right)\left(B_{n} \sqrt{r} K_{n+\frac{1}{2}}(\alpha r)\right)\right] .
$$

where $K_{a}(x)$ is the Modified Bessel Function of the 2nd kind of order $a$. 
We then use separation of variables with the definition of the operator (4) to solve equation (23) for $\psi_{1}$ noting that (23) gives the particular solution of $\psi_{1}$. Hence we finally obtain

$$
\begin{gathered}
\psi_{1}=e^{i t}\left[\left(\int_{\mu}^{1} P_{0}(x) d x+e_{0}\right)\left(A_{0} \frac{\sqrt{r}}{\alpha^{2}} I_{\frac{1}{2}}(\alpha r)+B_{0} \frac{\sqrt{r}}{\alpha^{2}} K_{\frac{1}{2}}(\alpha r)+C_{0} r+D_{0}\right)+\right. \\
\left.\sum_{n=1}^{\infty}\left(\int_{\mu}^{1} P_{n}(x) d x\right)\left(A_{n} \frac{\sqrt{r}}{\alpha^{2}} I_{n+\frac{1}{2}}(\alpha r)+B_{n} \frac{\sqrt{r}}{\alpha^{2}} K_{n+\frac{1}{2}}(\alpha r)+C_{n} r^{n+1}+D_{n} r^{-n}\right)\right],
\end{gathered}
$$

where $\alpha=(1+i) \delta^{-1}, I_{a}(x)$ is the Modified Bessel Function of the 1st kind of order $a$, and $A_{n}, B_{n}, C_{n}, D_{n}$ are constants to be determined using the boundary conditions.

\subsection{Enforcing the boundary conditions}

Since $I_{n}(\alpha r)$ increases to infinity exponentially as $r \rightarrow \infty$, we first see that the boundary conditions at infinity impose that $A_{n}=0$ for all $n$ and similarly $C_{n}=0$ for $n>0$.

Secondly, singularities in the velocity profile should be removed. This is not a problem for $u_{r}$ but it is for $u_{\theta}$. Indeed, we have the scaling $u_{\theta} \sim(1-$ $\left.\mu^{2}\right)^{-\frac{1}{2}}\left(\int_{\mu}^{1} P_{n}(x) d x\right)$, which has formal singularities at $\mu= \pm 1$. Using the identity

$$
\int_{\mu}^{1} P_{n}(x) d x=\frac{\left(1-\mu^{2}\right) P_{n}^{\prime}(\mu)}{n(n+1)} \text { for } n \neq 0,
$$

we see that the singularities are removable for $n>0$ but not for $n=0$. Since $e_{0}$ provides only one degree of freedom, it can be used to remove at most one singularity. Therefore, in order to prevent any singularity in $u_{\theta}$ at $\mu= \pm 1$, it is required that $C_{0}=B_{0}=0$. The only term remaining in $\psi_{1}$ containing $e_{0}$ is proportional to $e_{0} D_{0}$, which is an arbitrary constant and hence $e_{0}$ can be set to 0 .

With these results, $\psi_{1}$ is reduced to

$$
\psi_{1}=e^{i t}\left[\left(D_{0} \int_{\mu}^{1} P_{0}(x) d x\right)+\sum_{n=1}^{\infty}\left(\int_{\mu}^{1} P_{n}(x) d x\right)\left(B_{n} \frac{\sqrt{r}}{\alpha^{2}} K_{n+\frac{1}{2}}(\alpha r)+D_{n} r^{-n}\right)\right] .
$$

Applying the two first-order boundary conditions, (20) and (21), finally allows the determination of the remaining constants $B_{n}, D_{n}$ and $D_{0}$. For ease of expansion later, a Bessel function factor evaluated at $\alpha$ has been left formally 
in the definition of the constants giving us

$$
\begin{aligned}
& B_{n}=\frac{-\left(W_{n}+n V_{n}\right)}{K_{n+\frac{1}{2}}(\alpha)}\left(\frac{(1+i)}{\delta}+n+\delta \frac{(i-1) n^{2}}{4}\right)+O\left(\frac{\delta^{2}}{K_{n+\frac{1}{2}}(\alpha)}\right) \text { for } n \geq 1 \\
& D_{n}=V_{n}+\frac{\delta(1-i)\left(W_{n}+n V_{n}\right)}{2}-\frac{i n\left(W_{n}+n V_{n}\right) \delta^{2}}{2}+O\left(\delta^{3}\right) \text { for } n \geq 1 \\
& D_{0}=V_{0} .
\end{aligned}
$$

For simplicity of notation in what follows, we define $W_{0}=0$ so that (27) and similarly (28) remain valid for $n=0$.

\section{Second-order asymptotic solution}

At $O\left(\epsilon^{2}\right)$, a net fluid motion will arise and from this we will obtain the streaming flow. The first-order solution determines the explicit second order boundary conditions (\$4.1) and provides a non-zero forcing term $(\S 4.2)$ at $O\left(\epsilon^{2}\right)$. Unfortunately, the governing equation with this volume forcing is too complex to solve explicitly analytically. We will thus employ asymptotic matching in order to solve for the flow inside the boundary layer of size $\eta=1+\delta r(\S 4.3)$ and for the flow in the far field ( $\S 4.4)$ where exponential decay of this forcing leads to a Stokes flow. Upon matching these two solutions (§4.5) the outer solution will give access to the Eulerian streaming flow around the body.

\subsection{Second-order boundary conditions}

Equations (26)-(29) give the full solution for $\psi_{1}$. Using (15) and (16) we can now time-average the boundary conditions at order $\epsilon^{2}$. Note that the product of two terms of the form $f e^{i t}$ and $g e^{i t}$ time average to $f \bar{g} / 2$ or $\bar{f} g / 2$ where the real part is assumed and overbars denote complex conjugates. Simplifying the $\mu$ dependence of the result to a sum over the appropriate basis functions, i.e. $\left(1-\mu^{2}\right)^{-\frac{1}{2}} \int_{\mu}^{1} P_{n}(x) d x$ for $u_{\theta}$ and $P_{n}(\mu)$ for $u_{r}$ and using the classical identities

$$
\begin{aligned}
x P_{n}^{\prime}(x) & =P_{n+1}^{\prime}(x)-(n+1) P_{n}(x), \\
P_{n+1}^{\prime}(x) & =P_{n-1}^{\prime}(x)+(2 n+1) P_{n}(x),
\end{aligned}
$$

we explicitly obtain time-averaged boundary conditions

$$
\begin{aligned}
\left.\left\langle u_{r}^{(2)}\right\rangle\right|_{r=1}=\sum_{k=1}^{\infty}\{ & \frac{i}{2}\left[2 V_{0} \bar{V}_{k}-\sum_{n=0}^{\infty} \sum_{m=1}^{\infty}\left(W_{m}-2 V_{m}\right) \bar{V}_{n} g_{k n m}\right. \\
& \left.\left.+\sum_{n=1}^{\infty} \sum_{m=1}^{\infty} \frac{W_{m}\left(\bar{V}_{n} n(n+1)-\bar{W}_{n}\right)}{m n(n+1)(m+1)} f_{k n m}\right]\right\} P_{k}(\mu),
\end{aligned}
$$


and

$$
\begin{aligned}
\left.\left\langle u_{\theta}^{(2)}\right\rangle\right|_{r=1} & =\sum_{k=1}^{\infty}\left\{\sum _ { n = 0 } ^ { \infty } \sum _ { m = 1 } ^ { \infty } a _ { k n m } \frac { i } { 2 } \left[2 V_{n} \bar{W}_{m}-\bar{V}_{n}\left(\left(W_{m}+m V_{m}\right) \frac{(1+i)}{\delta}\right.\right.\right. \\
& \left.\left.\left.+\left((m+1) W_{m}-m V_{m}\right)\right)-\bar{W}_{m}\left(W_{n}-\sum_{j=1}^{\infty} \frac{C_{n j}}{j(j+1)} W_{j}\right)\right]\right\}\left(\frac{\int_{\mu}^{1} P_{k}(x) d x}{\left(1-\mu^{2}\right)^{\frac{1}{2}}}\right)
\end{aligned}
$$

where we have used triangular brackets to indicate time-averaging.

In these equations, the series of coefficients $C_{n j}, a_{k n m}, f_{k n m}$ and $g_{k n m}$ are defined by

$$
C_{n j}= \begin{cases}0 & \text { if }(j<n) \text { or }(n \text { and } j \text { have different parity }) \\ n & \text { if } j=n \\ (2 n+1) & \text { otherwise }\end{cases}
$$

and

$$
\begin{aligned}
P_{n}(\mu)\left(\int_{\mu}^{1} P_{m}(x) d x\right) & =\sum_{k=1}^{\infty} a_{k n m}\left(\int_{\mu}^{1} P_{k}(x) d x\right), \\
P_{n}^{1}(\mu) P_{m}^{1}(\mu) & =\sum_{k=0}^{\infty} f_{k n m} P_{k}(\mu) \\
P_{n}(\mu) P_{m}(\mu) & =\sum_{k=0}^{\infty} g_{k n m} P_{k}(\mu)
\end{aligned}
$$

and where $P_{n}^{1}(\mu)$ is the Associated Legendre Polynomial of degree $n$ and order 1. Recall that Associated Legendre Polynomials of degree $n$ and order $m$ are defined by

$$
P_{n}^{m}(x)=\left(1-x^{2}\right)^{\frac{m}{2}} \frac{d^{m} P_{n}(x)}{d x^{m}}
$$

and have the useful orthogonality property

$$
\int_{-1}^{1} P_{n}^{m}(x) P_{n^{\prime}}^{m^{\prime}}(x) d x=\frac{2}{(2 n+1)} \frac{(n+m) !}{(n-m) !} \delta_{n n^{\prime}} \delta_{m m^{\prime}}
$$

Using the orthogonality property together with (25), the formulae for $a_{k n m}$ (35), $f_{k n m}(36)$ and $g_{k n m},(37)$ can be rearranged into a more useful form to calculate their numerical value

$$
\begin{aligned}
a_{k n m} & =\frac{k(k+1)}{m(m+1)} \frac{\int_{-1}^{1} P_{n}(x) P_{m}^{1}(x) P_{k}^{1}(x) d x}{\int_{-1}^{1} P_{k}^{1}(x) P_{k}^{1}(x) d x}=\frac{(2 k+1)}{2 m(m+1)} \int_{-1}^{1} P_{n}(x) P_{m}^{1}(x) P_{k}^{1}(x)(d .6) \\
f_{k n m} & =\frac{(2 k+1)}{2} \int_{-1}^{1} P_{n}^{1}(x) P_{m}^{1}(x) P_{k}(x) d x \\
g_{k n m} & =\frac{(2 k+1)}{2} \int_{-1}^{1} P_{k}(x) P_{n}(x) P_{m}(x) d x
\end{aligned}
$$


$a_{k n m}, f_{k n m}$ and $g_{k n m}$ are Gaunt Coefficients, which have been extensively studied due to their appearance in theoretical physics. Gaunt's formula [44] for the triple product integral and fast numerical algorithms [45] exist to evaluate such coefficients.

\subsection{Nonlinear Forcing}

The governing equation at order $\epsilon^{2}$ is given by

$$
\frac{\delta^{2}}{2}\left(D^{4} \psi_{2}\right)-\frac{\partial\left(D^{2} \psi_{2}\right)}{\partial t}=\frac{1}{r^{2}}\left(\frac{\partial\left(\psi_{1}, D^{2} \psi_{1}\right)}{\partial(r, \mu)}+2 L \psi_{1} D \psi_{1}\right) .
$$

Time averaging equation (43) leads to

$$
\frac{\delta^{2}}{2} D^{4}\left\langle\psi_{2}\right\rangle=\frac{1}{r^{2}}\left\langle\frac{\partial\left(\psi_{1}, D^{2} \psi_{1}\right)}{\partial(r, \mu)}+2 L \psi_{1} D \psi_{1}\right\rangle .
$$

A general second order solution that is valid throughout the domain cannot be found due to the complexity of the right-hand side of equation (44). However, for small values of $\delta$, solutions can be found separately within the viscous boundary layer and in the far field, and they can be asymptotically matched to provide a full outer solution. This is the method we will be using in this paper. In either case, an indication of the form of the non-linear forcing is required. The term $D^{2} \psi_{1}$ is given in equation (23). We also have

$$
\begin{aligned}
L \psi_{1}= & e^{i t}\left[\sum_{n=1}^{\infty}\left(\frac{\mu P_{n}^{\prime}(\mu)}{n(n+1)}\right)\left(\frac{B_{n}}{2 \alpha^{2} \sqrt{r}} K_{n+\frac{1}{2}}(\alpha r)+\frac{B_{n} \sqrt{r}}{\alpha} K_{n+\frac{1}{2}}^{\prime}(\alpha r)-D_{n} n r^{-(n+1)}\right)\right. \\
& \left.-\frac{D_{0} P_{0}(\mu)}{r}-\sum_{n=1}^{\infty}\left(P_{n}(\mu)\right)\left(\frac{B_{n}}{\sqrt{r} \alpha^{2}} K_{n+\frac{1}{2}}(\alpha r)+D_{n} r^{-(n+1)}\right)\right], \quad(45)
\end{aligned}
$$

and

$$
\begin{gathered}
\left\langle\frac{\partial\left(\psi_{1}, D^{2} \psi_{1}\right)}{\partial(r, \mu)}\right\rangle=-\frac{1}{2} \sum_{n=0}^{\infty} \sum_{m=1}^{\infty} P_{n}(\mu)\left(\int_{\mu}^{1} P_{m}(x) d x\right)\left(\frac{\bar{B}_{n} B_{m}}{\alpha^{2}} \bar{K}_{n+\frac{1}{2}}(\alpha r) K_{m+\frac{1}{2}}(\alpha r)\right. \\
+2 \frac{\bar{B}_{n} B_{m} r}{\alpha} \bar{K}_{n+\frac{1}{2}}(\alpha r) K_{m+\frac{1}{2}}^{\prime}(\alpha r)-D_{m} \bar{B}_{n} m r^{-\left(m+\frac{1}{2}\right)} \bar{K}_{n+\frac{1}{2}}(\alpha r) \\
\left.-\frac{B_{m} \bar{D}_{n}}{2} K_{m+\frac{1}{2}}(\alpha r) r^{-\left(n+\frac{1}{2}\right)}-B_{m} \bar{D}_{n} \alpha r^{-\left(n-\frac{1}{2}\right)} K_{m+\frac{1}{2}}^{\prime}(\alpha r)\right)
\end{gathered}
$$

Using the coefficient $a_{k n m}$ defined in (35), equation (46) can then be transformed to the appropriate (integral polynomial) basis. Similarly the quantity given by $2 \times(45) \times(23)$ can have its basis transformed using $a_{k n m}$ and $C_{n j}$ 
defined in (34). These two quantities can then be added which gives the total non-linear forcing as

$$
\begin{gathered}
\left\langle 2 L \psi_{1} D^{2} \psi_{1}+\frac{\partial\left(\psi_{1}, D^{2} \psi_{1}\right)}{\partial(r, \mu)}\right\rangle= \\
\sum_{k=1}^{\infty}\left(\int_{\mu}^{1} P_{k}(x) d x\right)\left[\sum _ { n = 0 } ^ { \infty } \sum _ { m = 1 } ^ { \infty } a _ { k n m } \left(\frac{\bar{B}_{n} B_{m}}{2 \alpha^{2}} \bar{K}_{n+\frac{1}{2}}(\alpha r) K_{m+\frac{1}{2}}(\alpha r)\right.\right. \\
-\frac{3}{4} \bar{D}_{n} B_{m} r^{-\left(n+\frac{1}{2}\right)} K_{m+\frac{1}{2}}(\alpha r)-\frac{\bar{B}_{n} B_{m} r}{\alpha} \bar{K}_{n+\frac{1}{2}}(\alpha r) K_{m+\frac{1}{2}}^{\prime}(\alpha r)+\frac{1}{2} D_{m} \bar{B}_{n} m r^{-\left(m+\frac{1}{2}\right)} \bar{K}_{n+\frac{1}{2}}(\alpha r) \\
+\frac{1}{2} B_{m} \bar{D}_{n} \alpha r^{-\left(n-\frac{1}{2}\right)} K_{m+\frac{1}{2}}^{\prime}(\alpha r)+\sum_{j=1}^{\infty}\left(\frac{C_{n j}}{j(j+1)}\right)\left(-\frac{\bar{B}_{j} B_{m}}{2 \alpha^{2}} \bar{K}_{j+\frac{1}{2}}(\alpha r) K_{m+\frac{1}{2}}(\alpha r)\right. \\
\left.\left.\left.+\frac{\bar{B}_{j} B_{m} r}{\bar{\alpha}} K_{m+\frac{1}{2}}(\alpha r) \bar{K}_{j+\frac{1}{2}}^{\prime}(\alpha r)-\bar{D}_{j} j r^{-\left(j+\frac{1}{2}\right)} K_{m+\frac{1}{2}}(\alpha r) B_{m}\right)\right)\right] .
\end{gathered}
$$

4.3 Solution inside the boundary layer

The rest boundary is located at $r=1$ with a boundary layer of size $\delta$. We thus define an inner variable $\eta$ related to $r$ by $r=1+\delta \eta$. The boundary layer is small, $\delta \ll 1$, and within the boundary layer the inner variable $\eta$ varies from 0 to 1 . We now write the second-order equation in terms of $\eta$ and expand in ascending powers of $\delta$.

First consider expanding the right-hand side of equation (44), i.e. equation (47) divided by $r^{2}$. When the first-order boundary conditions were applied above, we obtained in equations (27)-(29) Taylor expansions of the coefficients $D_{k}$ and $B_{k}$ in terms of $\delta$. The powers of $r$ can also be Taylor expanded about $r=1$ to also obtain a powers series in $\delta$.

However the Taylor expansions of the Bessel Functions have to be done more carefully. A useful identity [46] for expanding the Bessel Function is that, for non-negative integer $n$ we have

$$
K_{n+\frac{1}{2}}(z)=\sqrt{\frac{\pi}{2}} \frac{e^{-z}}{\sqrt{z}} \sum_{j=0}^{n} \frac{(j+n) !}{(n-j) ! j !}(2 z)^{-j} .
$$

Next, notice that when the expansions for $D_{k}$ and $B_{k}$ are substituted into (44), the Bessel functions always appear in ratios of the form

$$
\frac{\hat{K}_{n+\frac{1}{2}}(\alpha r)}{K_{n+\frac{1}{2}}(\alpha)}
$$

where $\hat{K}$ represents a derivative or complex conjugate of the Bessel Function. As such, taking $f(r)$ as the appropriate power series in $r$ gives

$$
\frac{\hat{K}_{j+\frac{1}{2}}(\alpha r)}{K_{j+\frac{1}{2}}(\alpha)}=e^{-\tilde{\alpha}(r-1)} f(r)=e^{-(1 \pm i) \eta} f(1+\delta \eta),
$$


where $\tilde{\alpha}$ can be $\alpha$ or its complex conjugate. The exponential part of the Bessel function can clearly not be Taylor expanded in powers of $\delta$. Hence to obtain the correct expansion, the power series part of the Bessel Function should be Taylor expanded in powers of $\delta$ and the negative exponential part should only have the appropriate $\eta$ substitution carried out.

Upon completion of the substitution and expansion in $\delta$, we obtain inside the boundary layer

$$
\begin{gathered}
\frac{1}{r^{2}}\left\langle 2 L \psi_{1} D^{2} \psi_{1}+\frac{\partial\left(\psi_{1}, D^{2} \psi_{1}\right)}{\partial(r, \mu)}\right\rangle=\sum_{k=1}^{\infty}\left(\int_{\mu}^{1} P_{k}(x) d x\right) \sum_{n=0}^{\infty} \sum_{m=1}^{\infty} a_{k n m} \times \\
\left\{\frac{3}{4}\left(W_{m}+m V_{m}\right) e^{-(1+i) \eta}\left(\frac{\bar{V}_{n}(1+i)}{\delta}\right)+\left(\bar{W}_{n}+n \bar{V}_{n}\right)\left(W_{m}+m V_{m}\right)\left(\frac{1-i}{\delta}\right) e^{-2 \eta}\right. \\
\quad-\frac{1}{2} m\left(\bar{V}_{n} n+\bar{W}_{n}\right)\left(V_{m} \frac{(1-i)}{\delta}\right) e^{-(1-i) \eta}+\frac{1}{2}\left(W_{m}+m V_{m}\right)\left[\frac{2 i \bar{V}_{n}}{\delta^{2}}+\right. \\
\left.\frac{1}{\delta}\left(-2 i n V_{n} \eta+\bar{V}_{n} m(1+i)-(1-i)\left(\bar{W}_{n}+n \bar{V}_{n}\right)+(i+1) \frac{\bar{V}_{n}}{2}-4 i V_{n} \eta\right)\right] e^{-(1+i) \eta} \\
+\sum_{j=1}^{\infty}\left(\frac{C_{n j}}{j(j+1)}\right)\left[-\left(\bar{W}_{j}+j \bar{V}_{j}\right)\left(W_{m}+m V_{m}\right) e^{-2 \eta}\left(\frac{(1+i)}{\delta}\right)\right. \\
\left.\left.+\left(W_{m}+m V_{m}\right) e^{-(1+i) \eta}\left(\frac{\bar{V}_{j} j(1+i)}{\delta}\right)\right]+O(1)\right\}
\end{gathered}
$$

Inside the boundary layer, the $D^{4}$ operator on the left hand side of (44) is asymptotically given by

$$
D^{4}=\frac{1}{\delta^{4}} \frac{\partial^{4}}{\partial \eta^{4}}+O\left(\delta^{-2}\right),
$$

and therefore (44) finally simplifies to

$$
\begin{gathered}
\left\langle\frac{\partial^{4} \psi_{2}^{i}}{\partial \eta^{4}}\right\rangle=\sum_{k=1}^{\infty}\left(\int_{\mu}^{1} P_{k}(x) d x\right) \sum_{n=0}^{\infty} \sum_{m=1}^{\infty} a_{k n m}\left\{\frac{3}{2} \delta\left(W_{m}+m V_{m}\right) e^{-(1+i) \eta} \bar{V}_{n}(1+i)\right. \\
+2 \delta\left(\bar{W}_{n}+n \bar{V}_{n}\right)\left(W_{m}+m V_{m}\right)(1-i) e^{-2 \eta}-\delta m\left(\bar{V}_{n} n+\bar{W}_{n}\right) V_{m}(1-i) e^{-(1-i) \eta} \\
+\left(W_{m}+m V_{m}\right)\left[\delta\left(-2 i n \bar{V}_{n} \eta+\bar{V}_{n} m(1+i)-(1-i)\left(\bar{W}_{n}+n \bar{V}_{n}\right)+(i+1) \frac{\bar{V}_{n}}{2}-4 i \bar{V}_{n} \eta\right)\right. \\
\left.+2 i \bar{V}_{n}\right] e^{-(1+i) \eta}+\sum_{j=1}^{\infty}\left(\frac{C_{n j}}{j(j+1)}\right)\left[-2 \delta\left(\bar{W}_{j}+j \bar{V}_{j}\right)\left(W_{m}+m V_{m}\right) e^{-2 \eta}(1+i)\right. \\
\left.\left.+2 \delta\left(W_{m}+m V_{m}\right) e^{-(1+i) \eta}\left(\bar{V}_{j} j(1+i)\right)\right]\right\}+O(1) .
\end{gathered}
$$

where $\psi_{2}^{i}$ is the second order stream function inside the boundary layer. 
Using the elementary indefinite integrals (where $c$ is the constant of integration)

$$
\begin{gathered}
\int_{y} \int_{\tilde{\tilde{\tilde{y}}}} \int_{\tilde{\tilde{y}}} \int_{\tilde{y}} e^{a x} d x d \tilde{y} d \tilde{\tilde{y}} d \tilde{\tilde{y}}=\frac{e^{a y}}{a^{4}}+c \\
\int_{y} \int_{\tilde{\tilde{y}}} \int_{\tilde{\tilde{y}}} \int_{\tilde{y}} x e^{a x} d x d \tilde{y} d \tilde{\tilde{y}} d \tilde{\tilde{y}}=\frac{y e^{a y}}{a^{4}}-\frac{4 e^{a y}}{a^{5}}+c
\end{gathered}
$$

allows us to integrate equation (53) explicitly, leading to the general inner solution

$$
\begin{aligned}
& \left\langle\psi_{2}^{i}\right\rangle=\sum_{k=1}^{\infty}\left\{( \int _ { \mu } ^ { 1 } P _ { k } ( x ) d x ) \sum _ { n = 0 } ^ { \infty } \sum _ { m = 1 } ^ { \infty } a _ { k n m } \left[-\frac{3}{8}\left(W_{m}+m V_{m}\right) e^{-(1+i) \eta} \bar{V}_{n}(1+i) \delta\right.\right. \\
& +\delta\left(\bar{W}_{n}+n \bar{V}_{n}\right)\left(W_{m}+m V_{m}\right) \frac{(1-i)}{8} e^{-2 \eta}+m\left(\bar{V}_{n} n+\bar{W}_{n}\right) V_{m} \frac{(1-i)}{4} \delta e^{-(1-i) \eta} \\
& -\left(W_{m}+m V_{m}\right)\left\{\frac { \delta } { 4 } \left[-2 i(n+2) \bar{V}_{n}(\eta+2(1-i))+\bar{V}_{n} m(1+i)-(1-i)\left(\bar{W}_{n}+n \bar{V}_{n}\right)\right.\right. \\
& \left.\left.+(i+1) \frac{\bar{V}_{n}}{2}\right]+\frac{i \bar{V}_{n}}{2}\right\} e^{-(1+i) \eta}+\sum_{j=1}^{\infty}\left(\frac{C_{n j}}{j(j+1)}\right)\left(-\left(\bar{W}_{j}+j \bar{V}_{j}\right)\left(W_{m}+m V_{m}\right) e^{-2 \eta} \frac{(1+i)}{8} \delta\right. \\
& \left.\left.\left.-\delta\left(W_{m}+m V_{m}\right) \frac{e^{-(1+i) \eta}}{2} \bar{V}_{j} j(1+i)\right)+O\left(\delta^{2}\right)\right]+\left(L_{k}+M_{k} \eta+N_{k} \eta^{2}+Q_{k} \eta^{3}\right)\right\},
\end{aligned}
$$

where $L_{k}, M_{k}, N_{k}$ and $Q_{k}$ are constants of integration to be determined. Of those, two will be determined by enforcing the two second-order boundary conditions (32) and (33), namely $L_{k}$ and $M_{k}$. Since these boundary conditions must hold for all $-1 \leq \mu \leq 1$, the coefficient of each basis function (i.e. $P_{n}(\mu)$ or $\left.\int_{\mu}^{1} P_{n}(x) d x\right)$ must obey these boundary conditions term by term, giving a countably infinite number of equations with solution

$$
\begin{aligned}
L_{k}= & \Re \frac{i}{2}\left[\sum_{n=0}^{\infty} \sum_{m=1}^{\infty} a_{k n m}\left(W_{m}+m V_{m}\right) \bar{V}_{n}+2 V_{0} \bar{V}_{k}-\sum_{n=0}^{\infty} \sum_{m=1}^{\infty}\left(W_{m}-2 V_{m}\right) \bar{V}_{n} g_{k n m}\right. \\
& \left.\left.+\sum_{n=1}^{\infty} \sum_{m=1}^{\infty} \frac{W_{m}\left(\bar{V}_{n} n(n+1)-\bar{W}_{n}\right)}{m n(n+1)(m+1)} f_{k n m}\right]\right\}+O(\delta), \quad k>0 \\
M_{k}= & \delta\left(\sum _ { n = 0 } ^ { \infty } \sum _ { m = 1 } ^ { \infty } a _ { k n m } \left\{\frac{-i m V_{m}\left(\bar{W}_{n}+n \bar{V}_{n}\right)}{2}+\frac{i \bar{V}_{n}}{2}\left(W_{m}+m V_{m}\right)(4+3 n-m)\right.\right. \\
+ & \frac{(3-i)}{4}\left(\bar{W}_{n}+n \bar{V}_{n}\right)\left(W_{m}+m V_{m}\right)+(m+3) \frac{i}{2} \bar{V}_{n} W_{m}-m \frac{i}{2} \bar{V}_{n} V_{m} \\
+ & \frac{i}{2} \bar{W}_{m} W_{n}-\sum_{j=1}^{\infty}\left(\frac{C_{n j}}{j(j+1)}\right)\left[\left(\bar{W}_{j}+j \bar{V}_{j}\right)\left(W_{m}+m V_{m}\right)\left(\frac{1+i}{4}\right)\right. \\
& \left.\left.\left.+i j \bar{V}_{j}\left(W_{m}+m V_{m}\right)+\frac{i}{2} \bar{W}_{m} W_{j}\right]\right\}\right)+O\left(\delta^{2}\right), \quad k>0 .
\end{aligned}
$$


Asymptotic matching will then determine the values of the remaining coefficients.

\subsection{Solution Outside the Boundary Layer}

Looking at equation (47), we see that all the terms in the non-linear forcing are multiples of Modified Bessel Functions of the second kind or their derivatives. As such, this forcing decays away exponentially fast as $r \rightarrow \infty$ and can be neglected outside the boundary layer. Note that this is equivalent to taking a Stokes, and not Oseen, approximation in the far field. Since the Stokes terms have power law decay rather than exponential decay, all of the corrections to the Stokes solution provided by an Oseen approximation will be of smaller order than infinitely many terms in our Stokes solution. Therefore in this region we have a Stokes flow and the governing equation (for the outer streamfunction $\left.\psi_{2}^{o}\right)$ is

$$
D^{2} D^{2}\left\langle\psi_{2}^{o}\right\rangle=0
$$

with exponentially small errors.

Given the form of the inner solution (56), and anticipating the asymptotic matching, we can look for the outer solution with a known $\mu$ dependence as

$$
\left\langle\psi_{2}^{o}\right\rangle=\sum_{n=1}^{\infty} f_{n}(r)\left(\int_{\mu}^{1} P_{n}(x) d x\right)+f_{0}(r)\left(\int_{\mu}^{1} P_{0}(x) d x+e_{0}\right) .
$$

The value of $\left\langle D^{2} \psi_{2}^{o}\right\rangle$ can be found by differentiating (60) but also by solving (59) for $\left\langle D^{2} \psi_{2}^{o}\right\rangle$ using separation of variables. Equating these gives a second order differential equation for $f$ with power-law solutions. The general outer solution is thus given by

$$
\begin{aligned}
\left\langle\psi_{2}^{o}\right\rangle= & \left(R_{0}+T_{0} r+Y_{0} r^{2}+S_{0} r^{3}\right)\left(\int_{\mu}^{1} P_{0}(x) d x+e_{0}\right) \\
& +\sum_{n=1}^{\infty}\left(R_{n} r^{n+3}+T_{n} r^{-n}+Y_{n} r^{n+1}+S_{n} r^{-(n-2)}\right)\left(\int_{\mu}^{1} P_{n}(x) d x\right)(61)
\end{aligned}
$$

Applying the boundary condition at infinity gives $R_{n}=Y_{n}=0$ and $S_{0}=Y_{0}=$ 0 . Furthermore, in order to avoid a singularity in $u_{\theta}$ at $\mu= \pm 1$ it is required $T_{0}=0$, and as $\left\langle\psi_{2}^{o}\right\rangle$ is a stream function it can be set that $e_{0}=0$ without affecting $u_{r}$ or $u_{\theta}$. Hence we obtain the outer solution as

$$
\left\langle\psi_{2}^{o}\right\rangle=R_{0}\left(\int_{\mu}^{1} P_{0}(x) d x\right)+\sum_{n=1}^{\infty}\left(T_{n} r^{-n}+S_{n} r^{-(n-2)}\right)\left(\int_{\mu}^{1} P_{n}(x) d x\right) .
$$




\subsection{Matching}

The final part of determining the solution for the flow consists of carrying out the asymptotic matching between the inner (56) and outer solutions (62). We first need to evaluate the inner solution, (56), in the limit $\eta \gg 1$ which, because of the negative exponentials, simplifies to

$$
\left\langle\psi_{2}^{i}\right\rangle=\sum_{k=1}^{\infty}\left(\int_{\mu}^{1} P_{k}(x) d x\right)\left(L_{k}+M_{k} \eta+N_{k} \eta^{2}+Q_{k} \eta^{3}\right) .
$$

The outer solution, (62), then needs to be evaluated in the limit $r \rightarrow 1$. Writing the outer solution in terms of the inner variable $\eta$ and Taylor expanding the expression about $\eta=0$ then gives

$$
\begin{aligned}
\left\langle\psi_{2}^{o}\right\rangle= & R_{0}\left(\int_{\mu}^{1} P_{0}(x) d x\right)+\sum_{n=1}^{\infty}\left(\int_{\mu}^{1} P_{n}(x) d x\right)\left[\left(T_{n}+S_{n}\right)-\left(n T_{n}+(n-2) S_{n}\right) \delta \eta\right. \\
& +\left(\frac{n(n+1)}{2} T_{n}+\frac{(n-2)(n-1)}{2} S_{n}\right) \delta^{2} \eta^{2} \\
& \left.-\left(\frac{n(n+1)(n+2)}{6} T_{n}+\frac{n(n-1)(n-2)}{6} S_{n}\right) \delta^{3} \eta^{3}+O\left(\eta^{4}\right)\right]
\end{aligned}
$$

Equating the two highest orders of $\eta$ gives

$$
\begin{aligned}
L_{k} & =T_{k}+S_{k} \quad(k \geq 1), \\
M_{k} & =-\left[k T_{k}+(k-2) S_{k}\right] \delta \quad(k \geq 1), \\
R_{0} & =0 .
\end{aligned}
$$

If we use $M_{k}^{(\delta)}$ to denote the $O(\delta)$ term of $M_{k}$, the outer constants are thus given by

$$
\begin{aligned}
T_{k} & =\frac{(2-k)}{2} L_{k}^{(1)}-\frac{M_{k}^{(\delta)}}{2 \delta}+O(\delta) \quad(k \geq 1), \\
S_{k} & =\frac{k}{2} L_{k}^{(1)}+\frac{M_{k}^{(\delta)}}{2 \delta}+O(\delta) \quad(k \geq 1) .
\end{aligned}
$$

Notice that $T_{k}$ and $S_{k}$ are now known so they can be used to determine $N_{k}$ and $Q_{k}$ by matching to third and fourth order; if one carries out this matching, one obtains that $N_{k}$ and $Q_{k}$ are $O\left(\delta^{2}\right)$ and $O\left(\delta^{3}\right)$ respectively.

\section{Lagrangian streaming}

The solution derived so far has focused on the Eulerian streaming, i.e. the timeaveraged Eulerian velocity field at a fixed position in the laboratory frame of reference. In order to compare with future experimental results tracking the motion of passive tracers in the flow, it is necessary to calculate the Lagrangian 
streaming instead. The difference between the Eulerian and the Lagrangian streaming is the so-called Stokes drift which arises because the Lagrangian particles are advected by the Eulerian velocities at all the positions the particles move through, and not just fixed positions in the laboratory frame, and thus velocity gradients need to be accounted for.

Longuet-Higgins $[47,38]$ showed that the streamfunction for the non-dimensional time averaged Stokes Drift $\bar{\varphi}_{S}$ at $O\left(\epsilon^{2}\right)$ is given by

$$
\left\langle\varphi_{S}\right\rangle=\overline{\frac{1}{r^{2}} \int \frac{\partial \psi_{1}}{\partial r} d t \frac{\partial \psi_{1}}{\partial \mu}}
$$

Ignoring exponentially-decaying terms, the outer solution for the Stokes Drift is thus

$$
\left\langle\varphi_{S}\right\rangle=-\frac{\epsilon^{2} i}{2 \omega} \sum_{k=1}^{\infty}\left(\int_{\mu}^{1} P_{k}(x) d x\right)\left(\sum_{n=0}^{\infty} \sum_{m=1}^{\infty} a_{k n m} m \bar{V}_{n} V_{m} r^{-(n+m+3)}\right)+O(\delta) .
$$

Adding this expression to the outer time-averaged Eulerian solution $\psi_{2}^{o}$ from equation (62) leads to the final expression for the outer leading order time averaged Lagrangian streaming as

$$
\left\langle\psi_{L}\right\rangle=\sum_{k=1}^{\infty}\left(T_{k} r^{-k}+S_{k} r^{-(k-2)}-\sum_{n=0}^{\infty} \sum_{m=1}^{\infty} Y_{k n m} r^{-(n+m+3)}\right)\left(\int_{\mu}^{1} P_{k}(x) d x\right),
$$

where

$$
Y_{k n m}=\Re\left(\frac{i a_{k n m} m \bar{V}_{n} V_{m}}{2}\right),
$$

and the coefficients $S_{k}$ and $T_{k}$ are given by

$$
\begin{aligned}
S_{k}= & \Re\left\{\sum _ { n = 0 } ^ { \infty } \sum _ { m = 1 } ^ { \infty } \left(a _ { k n m } \left\{\frac{i\left(W_{m}+m V_{m}\right) k \bar{V}_{n}}{4}\right.\right.\right. \\
& -\frac{i m V_{m}\left(\bar{W}_{n}+n \bar{V}_{n}\right)}{4}+\frac{i \bar{V}_{n}}{4}\left(W_{m}+m V_{m}\right)(4+3 n-m) \\
+ & \frac{(3-i)}{8}\left(\bar{W}_{n}+n \bar{V}_{n}\right)\left(W_{m}+m V_{m}\right)+(m+3) \frac{i}{4} \bar{V}_{n} W_{m}-m \frac{i}{4} \bar{V}_{n} V_{m} \\
+ & \frac{i}{4} \bar{W}_{m} W_{n}-\sum_{j=1}^{\infty}\left(\frac{C_{n j}}{j(j+1)}\right)\left[\left(\bar{W}_{j}+j \bar{V}_{j}\right)\left(W_{m}+m V_{m}\right)\left(\frac{1+i}{8}\right)\right. \\
& \left.\left.\left.+\frac{i}{2} j \bar{V}_{j}\left(W_{m}+m V_{m}\right)+\frac{i}{4} \bar{W}_{m} W_{j}\right]\right\}-\frac{i k}{4}\left(W_{m}-2 V_{m}\right) \bar{V}_{n} g_{k n m}\right) \\
+ & \left.\sum_{n=1}^{\infty} \sum_{m=1}^{\infty}\left[\frac{i k}{4} \frac{W_{m}\left(\bar{V}_{n} n(n+1)-\bar{W}_{n}\right)}{m n(n+1)(m+1)} f_{k n m}\right]+k V_{0} \bar{V}_{t} \frac{i}{2}\right\}+O(\delta),
\end{aligned}
$$


and

$$
\begin{aligned}
T_{k}=\Re\left\{\sum _ { n = 0 } ^ { \infty } \sum _ { m = 1 } ^ { \infty } \left(a _ { k n m } \left\{\left(W_{m}+m V_{m}\right)(2-k) \frac{i}{4} \bar{V}_{n}\right.\right.\right. \\
\quad+\frac{i m V_{m}\left(\bar{W}_{n}+n \bar{V}_{n}\right)}{4}-\frac{i \bar{V}_{n}}{4}\left(W_{m}+m V_{m}\right)(4+3 n-m) \\
\quad-\frac{(3-i)}{8}\left(\bar{W}_{n}+n \bar{V}_{n}\right)\left(W_{m}+m V_{m}\right)-(m+3) \frac{i}{4} \bar{V}_{n} W_{m}+m \frac{i}{4} \bar{V}_{n} V_{m} \\
\quad-\frac{i}{4} \bar{W}_{m} W_{n}+\sum_{j=1}^{\infty}\left(\frac{C_{n j}}{j(j+1)}\right)\left[\left(\bar{W}_{j}+j \bar{V}_{j}\right)\left(W_{m}+m V_{m}\right)\left(\frac{1+i}{8}\right)\right. \\
\left.\left.\left.+\frac{i}{2} j \bar{V}_{j}\left(W_{m}+m V_{m}\right)+\frac{i}{4} \bar{W}_{m} W_{j}\right]\right\}-\frac{i}{4}(2-k)\left(W_{m}-2 V_{m}\right) \bar{V}_{n} g_{k n m}\right) \\
\left.+\sum_{n=1}^{\infty} \sum_{m=1}^{\infty}\left[\frac{i}{4}(2-k) \frac{W_{m}\left(\bar{V}_{n} n(n+1)-\bar{W}_{n}\right)}{m n(n+1)(m+1)} f_{k n m}\right]+\frac{i}{2}(2-k) V_{0} \bar{V}_{k}\right\}+O(\delta),
\end{aligned}
$$

Since the inner solutions is only valid in a $\delta$ sized region about the spherical body this gives the Lagrangian solution in the bulk of the fluid.

We note that in the far field, the flow will be dominated by the slowest spatially decaying term. In the streamfunction (72) this is the $S_{1} r$ term. The velocities associated with this term decay as $\sim 1 / r$ and are associated with a net force acting on the fluid (stokeslet). This will be further discussed $\S 11$ in the context of force generation and propulsion.

\section{Special case: Squirming}

As discussed in the introduction, the squirmer model of low-Reynolds number swimming is a popular mathematical model to address the motion of nearly spherical ciliated cells (e.g. Opalina, Volvox) [15,13]. The array of deforming cilia is modelled as a continuous envelope where the effective tangential component is deemed far more significant than the radial component of motion. As such, the position of the surface can be modelled as fixed at its average position, and imposing steady velocities along it. Models of this form have been used to study nutrient uptake by microorganisms [48], interaction of microorganisms [49], optimal locomotion [50] and were recently generalised [16, 19]. Some versions of the squirming model do also allow a radial velocity to be applied through the fixed boundary as a model for a porous surface with normal jets of fluid through it [51].

The squirming approximation significantly increases the ease of theoretical calculations. However, and as expected, it has its limitations. A prescribed forcing through the boundary cannot be used to accurately model a moving boundary. If there is a non-zero radial velocity at the fixed boundary, the streaming flow will become one order of magnitude larger since the boundary 
conditions are no longer cancelling the leading-order term. In the very specific case of solely radial motion where all the radial modes are exactly $\pi / 4$ out of phase with each other the solution will be at the right order, but other important terms will still be missing from the streaming. This demonstrates the physical movement of the boundary, and hence the physical displacement of the fluid in that region, is as important as the prescribed velocities it is imparting to the fluid around it.

For angular motion alone, however, the squirmer streaming is identical to the full solution, demonstrating such an approximation is valid. Furthermore, in that situation the Lagrangian and Eulerian streamings are the same since the Stokes Drift depends only on the radial motion of the surface. We thus focus on the standard tangential squirmer model where there is no radial motion at leading-order and $V_{n}=0$ for all $n$. In that setup, the generated streaming is of the same form as has already been derived, (72), but the constants are much simpler with $Y_{k n m}=0$ and

$$
\begin{array}{r}
S_{k}=\Re\left\{\sum_{n=0}^{\infty} \sum_{m=1}^{\infty} a_{k n m}\left[\frac{3(1-i)}{8} \bar{W}_{n} W_{m}-\sum_{j=1}^{\infty}\left(\frac{C_{n j}}{j(j+1)}\right) \bar{W}_{j} W_{m}\left(\frac{1-i}{8}\right)\right]\right. \\
\left.+\sum_{n=1}^{\infty} \sum_{m=1}^{\infty}\left[-\frac{i k}{4} \frac{W_{m} \bar{W}_{n}}{m n(n+1)(m+1)} f_{k n m}\right]\right\}+O(\delta), \quad(76)
\end{array}
$$

and

$$
\begin{gathered}
T_{k}=\Re\left\{\sum_{n=0}^{\infty} \sum_{m=1}^{\infty} a_{k n m}\left[-\frac{3(1-i)}{8} \bar{W}_{n} W_{m}+\sum_{j=1}^{\infty}\left(\frac{C_{n j}}{j(j+1)}\right) \bar{W}_{j} W_{m}\left(\frac{1-i}{8}\right)\right]\right. \\
\left.+\sum_{n=1}^{\infty} \sum_{m=1}^{\infty}\left[-\frac{i}{4}(2-k) \frac{W_{m} \bar{W}_{n}}{m n(n+1)(m+1)} f_{k n m}\right]\right\}+O(\delta) .
\end{gathered}
$$

\section{Allowing Slip on the Boundary}

In previous sections we assumed that the fluid satisfied the no-slip boundary condition and thus exactly matched the motion of our deforming body. Slip can however be systematically incorporated into this model through a small change of boundary conditions. The general form of the solution remains the same but the constants of integration gain an extra contribution. This will then extend the model to include other spherical bodies, in particular bubbles.

In the case of no-slip, the motion of the boundary was described by its radial position, $R$, and angular position, $\Theta$. However instead $R$ and $\Theta$ can be interpreted as describing the motion of the fluid on the boundary of the spherical body. If we allow streaming at second order on the boundary, we can 
then write

$R=1-\epsilon \sum_{n=0}^{\infty} V_{n} P_{n}(\mu) e^{i\left(t+\frac{\pi}{2}\right)}+\epsilon^{2} \sum_{n=0}^{\infty} G_{n} P_{n}(\mu) g(t)+O\left(\epsilon^{3}\right)$,
$\Theta=\theta+\epsilon \sum_{n=1}^{\infty} W_{n}\left(\frac{\int_{\mu}^{1} P_{n}(x) d x}{\left(1-\mu^{2}\right)^{\frac{1}{2}}}\right) e^{i\left(t+\frac{\pi}{2}\right)}+\epsilon^{2} \sum_{n=0}^{\infty} F_{n}\left(\frac{\int_{\mu}^{1} P_{n}(x) d x}{\left(1-\mu^{2}\right)^{\frac{1}{2}}}\right) f(t)+O\left(\left(\epsilon^{3} 9\right)\right)$

which now has the extra second-order contributions where $F_{n}$ and $G_{n}$ are known constants determined by the motion of the spherical body and $f(t)$ and $g(t)$ are unknown functions of time.

The new definition of $R$ is equivalent to the previous one since the fluid and spherical body cannot encompass the same space. Therefore $V_{n}$ is still determined by the shape of the surface oscillation and there is still no net motion of the boundary, we thus have $G_{n}=0$ for all $n$. This new definition of $\Theta$ does allow net motion of the fluid along the body's surface, and the coefficients $W_{n}$ are then chosen so the appropriate surface boundary condition is obeyed at first order. Similarly the value of $F_{n}$ is determined by ensuring that this same surface boundary condition is obeyed at second order. As such $F_{n}$ may be nonzero and without loss of generality we assume that $\partial f / \partial t$ time averages to one.

The addition of the coefficients $F_{n}$ is a second-order contribution so $\psi_{1}$ is unchanged from (26). The form of the second-order inner solution (56) is unchanged with $L_{k}$ as in (57) but $M_{k}$ now has an extra $F_{k}$ contribution so we obtain the revised equation

$$
\begin{aligned}
M_{k}= & \delta\left(\sum _ { n = 0 } ^ { \infty } \sum _ { m = 1 } ^ { \infty } a _ { k n m } \left\{-i \frac{m}{2} V_{m}\left(\bar{W}_{n}+n \bar{V}_{n}\right)+\frac{i \bar{V}_{n}}{2}\left(W_{m}+m V_{m}\right)(4+3 n-m)\right.\right. \\
+ & \frac{(3-i)}{4}\left(\bar{W}_{n}+n \bar{V}_{n}\right)\left(W_{m}+m V_{m}\right)+(m+3) \frac{i}{2} \bar{V}_{n} W_{m}-m \frac{i}{2} \bar{V}_{n} V_{m} \\
+ & \frac{i}{2} \bar{W}_{m} W_{n}-\sum_{j=1}^{\infty}\left(\frac{C_{n j}}{j(j+1)}\right)\left[\left(\bar{W}_{j}+j \bar{V}_{j}\right)\left(W_{m}+m V_{m}\right)\left(\frac{1+i}{4}\right)\right. \\
& \left.\left.\left.+i j \bar{V}_{j}\left(W_{m}+m V_{m}\right)+\frac{i}{2} \bar{W}_{m} W_{j}\right]\right\}-F_{k}\right)+O\left(\delta^{2}\right) \text { for } k>0 .
\end{aligned}
$$

The new boundary condition considered, such as that of no angular stress along the surface of a bubble, would now be applied to this new second order inner solution allowing us to determine the value of the coefficients $F_{k}$.

Similarly the form of the outer solution (72) remains the same but through the asymptotic matching there is an extra contribution to its constants of 
integration so that the revised formulae for the $S_{k}$ and $T_{k}$ coefficients are now

$$
\begin{aligned}
S_{k}= & \Re\left\{\sum _ { n = 0 } ^ { \infty } \sum _ { m = 1 } ^ { \infty } \left[a _ { k n m } \left\{\frac{i\left(W_{m}+m V_{m}\right) k \bar{V}_{n}}{4}\right.\right.\right. \\
& -\frac{i m}{4} V_{m}\left(\bar{W}_{n}+n \bar{V}_{n}\right)+\frac{i \bar{V}_{n}}{4}\left(W_{m}+m V_{m}\right)(4+3 n-m) \\
+ & \frac{(3-i)}{8}\left(\bar{W}_{n}+n \bar{V}_{n}\right)\left(W_{m}+m V_{m}\right)+(m+3) \frac{i}{4} \bar{V}_{n} W_{m}-m \frac{i}{4} \bar{V}_{n} V_{m} \\
+ & \frac{i}{4} \bar{W}_{m} W_{n}-\sum_{j=1}^{\infty}\left(\frac{C_{n j}}{j(j+1)}\right)\left[\left(\bar{W}_{j}+j \bar{V}_{j}\right)\left(W_{m}+m V_{m}\right)\left(\frac{1+i}{8}\right)\right. \\
& \left.\left.\left.+\frac{i}{2} j \bar{V}_{j}\left(W_{m}+m V_{m}\right)+\frac{i}{4} \bar{W}_{m} W_{j}\right]\right\}-\frac{i k}{4}\left(W_{m}-2 V_{m}\right) \bar{V}_{n} g_{k n m}\right] \\
+ & \left.\sum_{n=1}^{\infty} \sum_{m=1}^{\infty}\left[\frac{i k}{4} \frac{W_{m}\left(\bar{V}_{n} n(n+1)-\bar{W}_{n}\right)}{m n(n+1)(m+1)} f_{k n m}\right]+k V_{0} \bar{V}_{k} \frac{i}{2}-\frac{F_{k}}{2}\right\}+O(\delta),
\end{aligned}
$$

and

$$
\begin{aligned}
T_{k}=\Re\left\{\sum _ { n = 0 } ^ { \infty } \sum _ { m = 1 } ^ { \infty } \left[a _ { k n m } \left\{\left(W_{m}+m V_{m}\right)(2-k) \frac{i}{4} \bar{V}_{n}\right.\right.\right. \\
\quad+\frac{i m}{4} V_{m}\left(\bar{W}_{n}+n \bar{V}_{n}\right)-\frac{i \bar{V}_{n}}{4}\left(W_{m}+m V_{m}\right)(4+3 n-m) \\
\quad-\frac{(3-i)}{8}\left(\bar{W}_{n}+n \bar{V}_{n}\right)\left(W_{m}+m V_{m}\right)-(m+3) \frac{i}{4} \bar{V}_{n} W_{m}+m \frac{i}{4} \bar{V}_{n} V_{m} \\
\quad-\frac{i}{4} \bar{W}_{m} W_{n}+\sum_{j=1}^{\infty}\left(\frac{C_{n j}}{j(j+1)}\right)\left[\left(\bar{W}_{j}+j \bar{V}_{j}\right)\left(W_{m}+m V_{m}\right)\left(\frac{1+i}{8}\right)\right. \\
\left.\left.\left.+\frac{i}{2} j \bar{V}_{j}\left(W_{m}+m V_{m}\right)+\frac{i}{4} \bar{W}_{m} W_{j}\right]\right\}-\frac{i}{4}(2-k)\left(W_{m}-2 V_{m}\right) \bar{V}_{n} g_{k n m}\right] \\
\left.+\sum_{n=1}^{\infty} \sum_{m=1}^{\infty}\left[\frac{i}{4}(2-k) \frac{W_{m}\left(\bar{V}_{n} n(n+1)-\bar{W}_{n}\right)}{m n(n+1)(m+1)} f_{k n m}\right]+\frac{i}{2}(2-k) V_{0} \bar{V}_{k}+\frac{F_{k}}{2}\right\}+O(\delta),
\end{aligned}
$$

with the coefficients $Y_{k n m}$ remaining the same as in (73). Naturally, through the asymptotic matching outlined above, as $T_{k}$ and $S_{k}$ have an extra contribution, the inner constants $N_{k}$ and $Q_{k}$ will also include a contribution proportional to the constants $F_{k}$. 


\section{Special case: Free surfaces}

In the case of an oscillating bubble, the extra boundary condition is that of no tangential stress on the bubble surface

$$
\boldsymbol{n} \cdot \boldsymbol{\sigma} \cdot \boldsymbol{t}=0, \quad \text { at } r=R, \theta=\Theta,
$$

where $\boldsymbol{t}$ is the tangent vector in the plane through the axis of axisymmetry, $\boldsymbol{n}$ is the normal vector and $\boldsymbol{\sigma}$ is the Newtonian stress tensor. There is still no penetration on the bubble surface and the shape of the bubble oscillation (via the coefficients $V_{n}$ ) is prescribed. Ensuring that the no-tangential stress conditions holds at first order and at second order (when time averaged) determines the values of $W_{n}$ and $F_{n}$. This calculation is quite involved and its details are given in Appendices $\mathrm{A}$ and $\mathrm{B}$ with the main results quoted in what follows.

\subsection{General case}

In this subsection we calculate the leading-order streaming provided the result is non-zero (see below). The generated streaming is of the same form as the one already derived, (72), with $Y_{k n m}$ defined as in (73) and $R_{0}=0$, with the difference that the constants $T_{k}$ and $S_{k}$ now take new values

$$
\begin{gathered}
T_{k}=\Re\left\{\frac{\left(1-k^{2}\right)}{(2 k+1)} i V_{0} \bar{V}_{k}+\sum_{n=1}^{\infty} \sum_{m=1}^{\infty} \frac{\left(k^{2}-k-1\right)(n+2)}{2(2 k+1)(n+1)(m+1)} f_{k n m} i \bar{V}_{n} V_{m}\right. \\
+\sum_{n=0}^{\infty} \sum_{m=1}^{\infty} \frac{3\left(1-k^{2}\right)}{2(2 k+1)} i V_{m} \bar{V}_{n} g_{k n m}+\sum_{n=0}^{\infty} \sum_{m=1}^{\infty} \frac{a_{k n m}}{2(2 k+1)}\left[m \left(n^{2}-4 n m-4 n\right.\right. \\
\left.\left.\left.+m^{2}-m-3\right) i \bar{V}_{n} V_{m}+\sum_{j=1}^{\infty}\left(\frac{C_{n j}}{(j+1)}\right)\left(m(3 m+5) i V_{m} \bar{V}_{j}\right)\right]\right\}+O(\delta),
\end{gathered}
$$

and

$$
\begin{gathered}
S_{k}=\Re\left\{\frac{k(k+2)}{(2 k+1)} i V_{0} \bar{V}_{k}-\sum_{n=1}^{\infty} \sum_{m=1}^{\infty} \frac{k(n+2)(2 k+4)}{4(n+1)(m+1)(2 k+1)} f_{k n m} i \bar{V}_{n} V_{m}\right. \\
+\sum_{n=0}^{\infty} \sum_{m=1}^{\infty} \frac{3 k(k+2)}{2(2 k+1)} i V_{m} \bar{V}_{n} g_{k n m}-\sum_{n=0}^{\infty} \sum_{m=1}^{\infty} \frac{a_{k n m}}{2(2 k+1)}\left[m \left(n^{2}-4 n m-4 n\right.\right. \\
\left.\left.\left.+m^{2}-m-3\right) i \bar{V}_{n} V_{m}+\sum_{j=1}^{\infty}\left(\frac{C_{n j}}{(j+1)}\right)\left(m(3 m+5) i V_{m} \bar{V}_{j}\right)\right]\right\}+O(\delta) .
\end{gathered}
$$

This solution is derived in detail in Appendix A. 
8.2 Special case - In-phase motion

If the $V_{n}$ coefficients are chosen such that the real part of $i V_{n} \bar{V}_{m}=0$ for all valued of $n$ and $m$ (i.e. all modes are in phase or $\pi$ out-of-phase with each other) then $T_{k}=S_{k}=Y_{k n m}=0$ and the steady streaming is identically zero at $O(1)$. This of course includes the case where only one mode is being forced. The net streaming in that case occurs at order $O(\delta)$. In order to determine this streaming the solution derived in $\S 2$ needs to be taken to third order, to give one more power of $\delta$, and then have the no-stress condition applied to it. The details for this calculation are in Appendix B. The generated streaming is still of the same form as has already been derived, (72) but with $Y_{k n m}=0$, $R_{0}=0$ and with the constants $T_{k}$ and $S_{k}$ now taking new values as

$$
\begin{aligned}
& T_{k}=\delta \Re\left\{\sum_{n=1}^{\infty} \sum_{m=1}^{\infty} \frac{\left(1-k^{2}\right)(n+2)}{2(2 k+1)(n+1)}\left(\bar{V}_{n} V_{m}\right) f_{k n m}-\sum_{n=0}^{\infty} \sum_{m=0}^{\infty} \frac{\left(1-k^{2}\right) m(m+2)}{2(2 k+1)}\left(V_{m} \bar{V}_{n}\right) g_{k n m}\right. \\
& +\sum_{n=0}^{\infty} \sum_{m=1}^{\infty} a_{k n m}\left[\left(\frac{n^{2}}{4}+\frac{9 n}{4}-\frac{5 m^{2}}{4}-\frac{5 m}{4}+1+\frac{1}{2} k^{2}+\frac{3}{2} k\right) \frac{m(m+2)}{2(2 k+1)} V_{m} \bar{V}_{n}\right. \\
& \left.\left.-\frac{n m(n+2)(2 m+1)}{2(2 k+1)} V_{m} \bar{V}_{n}+\sum_{j=1}^{\infty}\left(\frac{C_{n j}}{j(j+1)}\right)\left(-\frac{m j(2 m+j+6)}{2(2 k+1)} V_{m} \bar{V}_{j}\right)\right]\right\},
\end{aligned}
$$

and

$$
\begin{aligned}
& S_{k}=\delta \Re\left\{\sum_{n=1}^{\infty} \sum_{m=1}^{\infty} \frac{k(k+2)(n+2)}{2(n+1)(2 k+1)}\left(\bar{V}_{n} V_{m}\right) f_{k n m}-\sum_{n=0}^{\infty} \sum_{m=0}^{\infty} \frac{k(k+2) m(m+2)}{2(2 k+1)}\left(V_{m} \bar{V}_{n}\right) g_{k n m}\right. \\
& +\sum_{n=0}^{\infty} \sum_{m=1}^{\infty} a_{k n m}\left[-\left(\frac{n^{2}}{4}+\frac{9 n}{4}-\frac{5 m^{2}}{4}-\frac{5 m}{4}+\frac{1}{2} k(k-1)\right) \frac{m(m+2)}{2(2 k+1)} V_{m} \bar{V}_{n}\right. \\
& \left.\left.+\frac{n m(n+2)(2 m+1)}{2(2 k+1)} V_{m} \bar{V}_{n}+\sum_{j=1}^{\infty}\left(\frac{C_{n j}}{j(j+1)}\right)\left(\frac{m j(2 m+j+6)}{2(2 k+1)} V_{m} \bar{V}_{j}\right)\right]\right\}
\end{aligned}
$$

\subsection{Discussion}

Our results show thus that a bubble, for any in-phase oscillation of its shape, generates a streaming of $O(\delta)$ or lower, and therefore at least one order of magnitude weaker than that of a deformable no-slip surface (such as an elastic membrane) undergoing the same sequence of shape change. Thus the net flows generated by the angular velocities are of similar magnitude to those induced by the radial velocities and they cancel at leading order. 


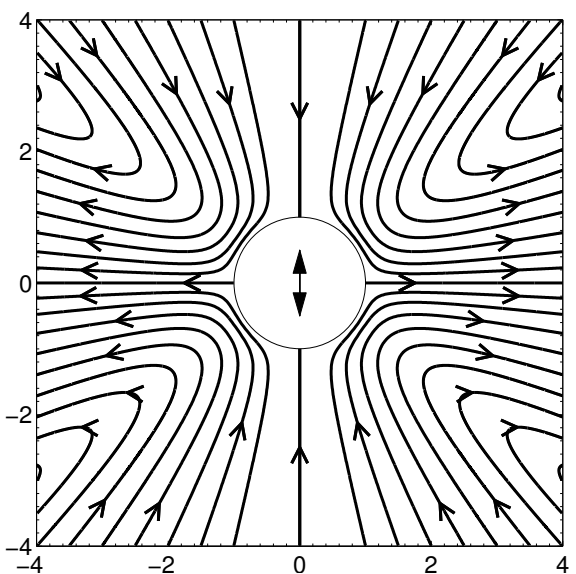

(a) Streaming from current model.

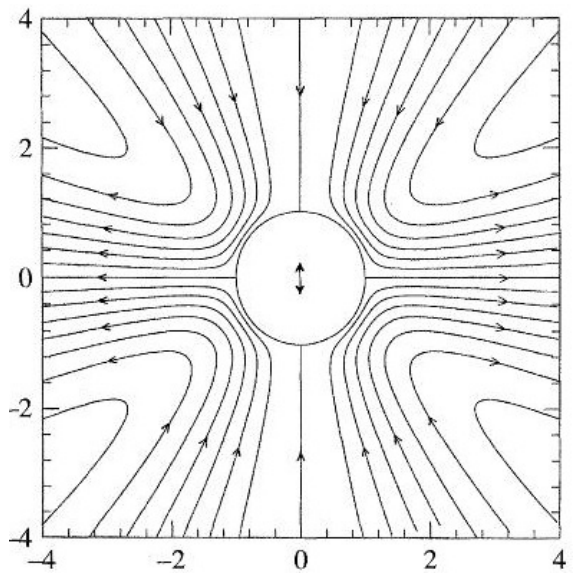

(b) Longuet-Higgins's streaming [38].

Fig. 2: Streaming, in the form of a stresslet, generated by the translational oscillations of a bubble

Longuet-Higgins observed that a bubble undergoing translational oscillation produced a force of $O(\delta)$, one order of magnitude less than the out-ofphase translational and pulsating oscillations which is $O(1)$ [38]. Our calculations allow us to generalise this result to all shape changes, and thus suggests that ensuring there are at least two modes of oscillation out of phase leads to stronger streaming flows.

For bubbles forced by external fields, this raises an interesting question of whether a resonance mode of oscillation, which is solely at one mode, would produce a weaker streaming flow than out-of-phase forcing which excites multiple modes. From a practical standpoint, microbubbles are often fixed to a wall, which enforces that the center of the bubble has to move and as such is naturally excited at a second mode.

\section{Comparison with past work}

Our calculations have allowed us to compute the streaming generated by any specified, fixed, oscillating spherical object (and in particular we solved for a bubble). Past work has characterized the streaming flow for simple shape oscillations of bubbles and rigid spheres, to which we can compare our model in order to validate it.

\subsection{Translating Bubble}

In the case of a bubble undergoing translational oscillations, we have $V_{1}=1$ and $V_{n}=0$ for $n \neq 1$, The angular boundary conditions $W_{n}$ and $F_{n}$ are 


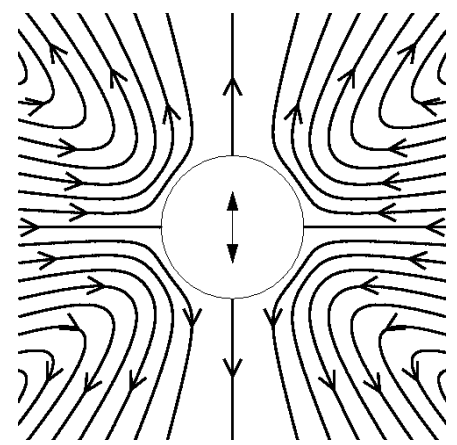

(a)

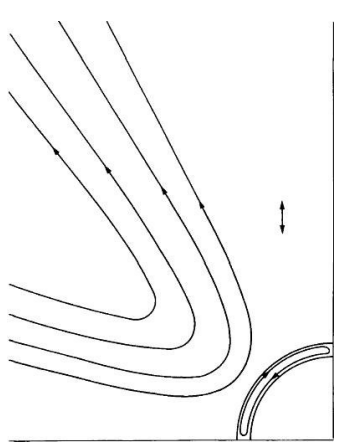

(b) Riley's streaming [36].

Fig. 3: Streaming, in the form of a stresslet, generated by the translational oscillations of a sphere

determined by the no stress boundary condition. This case was studied by Longuet-Higgins [38] and the solution we obtain here is identical to his, namely

$$
\left\langle\psi_{L}\right\rangle=\delta \frac{27}{20}\left(\frac{1}{r^{2}}-1\right) \int_{\mu}^{1} P_{2}(x) d x,
$$

as further illustrated in Figure 2. This streaming flow is a stresslet with fluid pulled in along the axis of oscillation and pushed out along the equator.

\subsection{Translating Sphere}

In the case of a solid sphere undergoing translational oscillations we have $V_{1}=1, V_{n}=0$ for $n \neq 1, W_{1}=2, W_{n}=0$ for $n \neq 1$, and $F_{n}=0$. This case was studied by Riley [36] and the streaming we obtain is identical to his solution, namely

$$
\left\langle\psi_{2}^{o}\right\rangle=-\frac{45}{16}\left(\frac{1}{r^{2}}-1\right) \int_{\mu}^{1} P_{2}(x),
$$

as further illustrated in Figure 3. Similarly to the oscillating bubble, this streaming flow is a stresslet but with opposite direction.

\subsection{Bubble Translating and Radially Oscillating}

Finally, in the case of a bubble undergoing radial and lateral oscillations only $V_{0}$ and $V_{1}$ can be non-zero. Then the pairs $W_{n}$ and $F_{n}$ are determined by the no stress boundary condition. We obtain $W_{1}=-V_{1}, W_{n}=0$ for $n \neq 1$, $F_{1}=4 i \bar{V}_{0} V_{1}$ and $F_{n}=0$ for $n \neq 1$. In this case there is a contribution from 
the Stokes Drift with one non-zero component of $Y, Y_{101}=i \bar{V}_{0} V_{1} / 2$ leading to the final Lagrangian streaming as

$$
\left\langle\psi_{L}\right\rangle=\Re\left(i \bar{V}_{0} V_{1}\right)\left(-\frac{1}{4 r}+\frac{r}{2}-\frac{1}{4 r^{4}}\right)\left(1-\mu^{2}\right),
$$

which matches the result of Longuet-Higgins [38] (note that in Ref. [38], $\Re\left(i \bar{V}_{0} V_{1}\right)$ is written as $\sin (\phi)$ with $\phi$ denoting the phase difference between modes 0 and 1). At leading order this streaming flow is a stokeslet, with direction parallel to the axis of axisymmetry, and with a sign determined by the phase difference between $V_{0}$ and $V_{1}$.

\section{Illustration of steady streaming and far-field behaviour}

With the calculations above, we can now illustrate the streaming patterns which can be obtained from surface oscillations. We consider a range of surface boundary conditions $\left(V_{n}\right.$ and $W_{n}$ ) and assume for simplicity that the surface streaming is zero $\left(F_{n}=0\right)$. The steady streaming flow splits naturally into two regions with different behaviours: the fluid motion close to the spherical body, which often contains recirculation regions, and the far-field behaviour which is dominated by the slowest decaying term in the velocity.

First we consider the streaming generated by a few simple surface shape oscillations. In Figure 4a we illustrate the streaming when only the mode $V_{1} \neq 0$ is being forced. In all cases, the flow being axisymmetric, we only need to display streamlines in the plane of symmetry to illustrate the whole flow. Here $S_{1}=0$ so the slowest decaying term is $S_{2} / r^{2}$ which produces the pattern of flow coming in in the equatorial plane of the spherical body and pushed away along the vertical axis (stresslet, see below). Furthermore, as there are few higher order terms this behaviour in fact dominates the flow throughout the domain.

Differences between the far-field flow and the fluid motion close to the body can be seen with higher modes. This is illustrated in Figure $4 \mathrm{~b}$ which shows the streaming generated by forcing mode $V_{2}$ only. On the edge of the figure the dynamics seen in figure $4 \mathrm{a}$ is apparent as the term $S_{2} / r^{2}$ is still dominant in the far field. However close to the spherical body, we see circulations zones which extend about one body diameter into the fluid. The number of these circulations regions increase as higher modes are being forced. A similar pattern is observed when only oscillating at one angular mode (i.e. $W_{n} \neq 0$ for one choice of $n$ ).

Let us now consider the behaviour in the far field. Far from the sphere, the steady streaming is dominated by the slowest decaying term. If $S_{1} \neq 0$ then the slowest decaying flow is the stokeslet with the velocities decaying as $S_{1} / r$. This produces a non-zero force along the axis of rotational symmetry and this gives a clear movement of flow parallel to this axis - either in the positive (Figure 4c) or negative direction (Figure $4 \mathrm{~d}$ ). If $S_{1}=0$ the body has no force acting on it, as seen in Figures $4 \mathrm{a}$ and $4 \mathrm{~b}$ where the symmetry of the system 


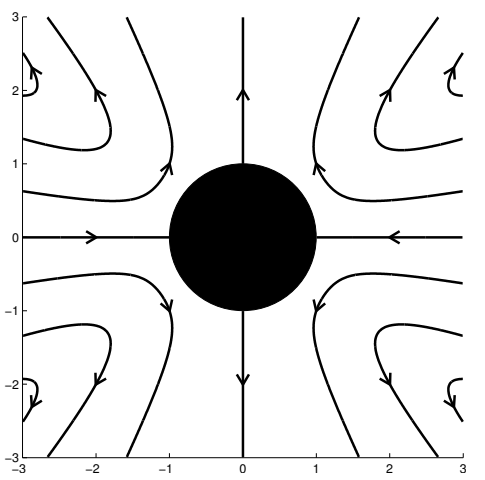

(a) Oscillating with $V_{1}=1$ only.

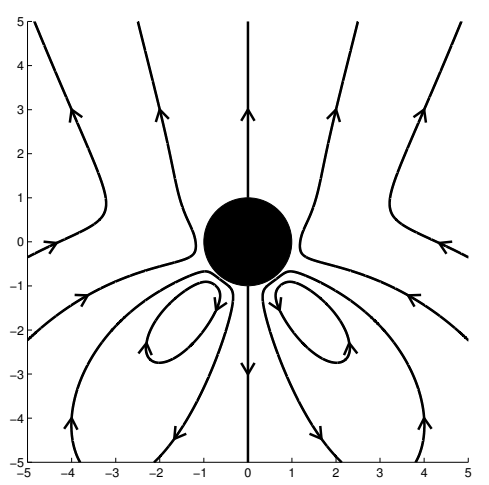

(c) Positive force: oscillating at two modes $V_{1}=\frac{1}{\sqrt{2}}, V_{2}=\frac{1}{\sqrt{2}}, W_{1}=\frac{-3}{\sqrt{2}}$ and $W_{2}=\frac{2}{\sqrt{2}}$.

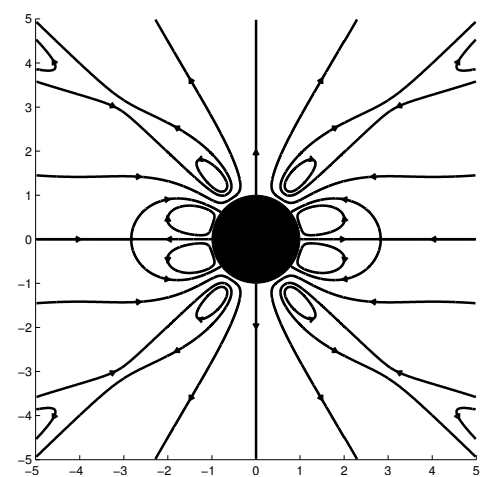

(b) Oscillating with $V_{2}=1$ only.

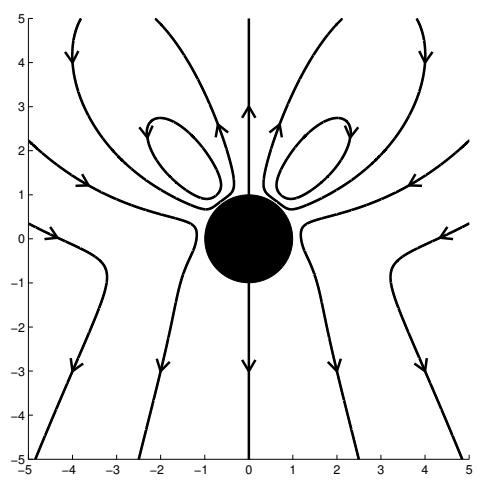

(d) Negative force : oscillating at two modes $V_{1}=\frac{1}{\sqrt{2}}$ and $V_{2}=\frac{1}{\sqrt{2}}$.

Fig. 4: Patterns of steady streaming for the first few surface oscillation modes.

prevents a net force from being induced. Generally, a net force is created only if two adjacent modes $\left\{V_{n}, V_{n+1}\right\}$ or $\left\{W_{n}, W_{n+1}\right\}$ are non-zero, as otherwise $a_{k n m}, g_{k n m}, f_{k n m}$ are all zero and the $a_{k n m} c_{n j}$ combinations cancel.

If the stokeslet coefficient, $S_{1}$, is zero the far field behaviour is dominated by a slower decaying term. In most cases it will be a stresslet with associated velocities decaying as $S_{2} / r^{2}$. This is the flow seen in Figure 4a. If in turn these terms are also zero then the far-field behaviour is dominated by the $\left(T_{k}+S_{k+2}\right) / r^{k+2}$ term for the lowest value of $k \geq 1$ which is non-zero.

Close to the spherical body, circulation regions will form. If there is a stokeslet, this term tends to dominate the flow and even close to the body circulation regions rarely appear. The one exception is close to the axis of symmetry where a pair of large circulations will sometimes form, either just above or just below the sphere (see Figure $4 \mathrm{~d}$ and $4 \mathrm{c}$ ). Since the flow is axisymmetric, this corresponds to a recirculation torus. 
Intuitively, one would expect that the shape of the volume physically displaced by the spherical body (set by the modes $V_{n}$ ) would have a significant effect on the features of the flow field. But as discussed in $\S 8$, the angular motion on the surface can produce a flow of similar magnitude, and in fact it can change the direction of the circulations and the streaming flow (and hence the direction of the body force applied to the spherical object) as demonstrated in Figures 4d and 4c. Another example of this, already seen in $§ 9$, displayed the streaming flow difference between a translating bubble (Figure 2) and a translating sphere in (Figure 3).

\section{Application: force generation and propulsion}

In our current setup, the sphere is held fixed, and the force exerted by the oscillations of the spherical body's surface on the fluid is computed. However, by Newton's law, an equal and opposite force is being applied to the spherical body from the fluid. If the spherical body is not held in place then this would cause it to move. Mathematically, a net motion of the body is necessarily a second-order effect as all leading-order effects are oscillatory and produce no net motion or forces. Hence allowing the body to move will only slightly modify our mathematical approach. In this section we characterise the force induced by a fixed body and then show how to carry the calculation in the case where the body is free to move.

\subsection{Force generation}

Due to the axisymmetry of the system a net force can only be exerted along the axis of rotational symmetry, taken to be $\mathbf{e}_{z}$ using traditional notation from spherical coordinates. We thus write $\boldsymbol{F}=F \mathbf{e}_{z}$.

The time averaged force on the spherical body is equal to the force across the boundary of our spherical object at $r=R$, i.e.

$$
\boldsymbol{F}=\left\langle\left.\int(\boldsymbol{\sigma} \cdot \boldsymbol{n})\right|_{r=R(\mu)} d S\right\rangle .
$$

This force must match the force across the boundary " $r=\infty$ " and thus

$$
\boldsymbol{F}=-\left\langle\left.\int(\boldsymbol{\sigma} \cdot \boldsymbol{n})\right|_{r=\infty} d S\right\rangle
$$

Across $r=\infty$, if the time average is taken then there will only be a contribution from the second order term as all first-order terms are oscillatory. Also, the region $r=\infty$ is now outside the boundary layer, where, due to the exponential decay of the non-linear terms, the time-averaged behaviour is a Stokes flow. 
Outside the boundary layer, the slowest decaying velocity is the $1 / r$ term. This velocity field at leading order in $r$ is

$$
\begin{aligned}
& u_{r}=S_{1} \frac{\cos \theta}{r}+O\left(r^{-3}\right), \\
& u_{\theta}=-S_{1} \frac{\sin \theta}{2 r}+O\left(r^{-3}\right) .
\end{aligned}
$$

This is the stokeslet discussed above. Indeed a non-dimensional stokeslet due to a force $\boldsymbol{F}$ applied at the origin induces a flow $\boldsymbol{U}$ with components

$$
U_{j}=\frac{F_{i}}{8 \pi}\left(\frac{\delta_{i j}}{r}+\frac{x_{i} x_{j}}{r^{3}}\right) .
$$

With a force in the $\mathbf{e}_{z}$ direction this becomes gives

$$
\begin{aligned}
U_{r} & =\frac{F}{4 \pi r} \cos \theta, \\
U_{\theta} & =-\frac{F}{8 \pi r} \sin \theta .
\end{aligned}
$$

Equating these two forms of the stokeslet shows the non-dimensional force exerted on the spherical body by the surrounding fluid is

$$
\boldsymbol{F}=-8 \pi \epsilon^{2} \frac{S_{1}}{2} \boldsymbol{e}_{z} .
$$

This force is the result of a dominant pressure field, thus the dimensional scaling for the pressure indicates the scaling for the force. The Navier-Stokes equations indicate that the pressure scales with time varying inertia so $p \sim$ $\rho U a \omega \sim \rho a^{2} \omega^{2}$ implying that the dimensional force is

$$
\boldsymbol{F}=-4 \pi \epsilon^{2} S_{1}\left(\rho a^{4} \omega^{2}\right) \boldsymbol{e}_{z} .
$$

\subsection{Force-free swimming}

If the spherical body is no longer held in place but is free to move, it will translate with an $O\left(\epsilon^{2}\right)$ velocity in the direction of this force. However, the constraint of force-free motion needs to be carefully enforced at both $O(\epsilon)$ and $O\left(\epsilon^{2}\right)$ and being free to move will in general also impact the first-order oscillatory motion.

\subsubsection{Force-free motion at $O(\epsilon)$}

At $O(\epsilon)$ the motion of the spherical body is completely determined by the constants $V_{n}$ and $W_{n}$. Up to now these coefficients could be chosen arbitrarily to represent any surface motion. The extra constraint of force-free motion will now restrict the allowed motion of the spherical body, therefore restricting choices of $V_{n}$ and $W_{n}$. 
Mathematically, force-free motion is written as

$$
\boldsymbol{F}=\left.\int(\boldsymbol{\sigma} \cdot \boldsymbol{n})\right|_{r=R(\mu, t)} d S=0 .
$$

The normal vector to the surface of the spherical body is

$$
\hat{\boldsymbol{n}}=\boldsymbol{e}_{r}-\frac{\partial R}{\partial \theta} \boldsymbol{e}_{\theta}+O\left(\epsilon^{2}\right)
$$

Knowing that the direction of $\mathbf{F}$ is in the $\mathbf{e}_{z}$ direction by symmetry, this becomes

$$
\boldsymbol{F}=\left[\left.\int\left(\sigma_{r r} \cos \theta-\sigma_{\theta r} \sin \theta\right)\right|_{r=1} d S+O\left(\epsilon^{2}\right)\right] \boldsymbol{e}_{z}
$$

This can then be non-dimensionalised and the integral expanded to give

$\boldsymbol{F}=\left\{\int_{0}^{2 \pi} \int_{0}^{\pi}\left[\left(-p+\delta^{2} \frac{\partial u_{r}}{\partial r}\right) \cos \theta-\frac{\delta^{2}}{2}\left(\frac{1}{r} \frac{\partial u_{r}}{\partial \theta}+\frac{\partial u_{\theta}}{\partial r}-\frac{u_{\theta}}{r}\right) \sin \theta\right] \sin \theta d \theta d \phi+O\left(\epsilon^{2}\right)\right\} \boldsymbol{e}_{z}$.

The first-order pressure can be calculated by substituting the first-order solution for $\psi_{1},(26)$, into the Navier Stokes equation to obtain

$$
p=e^{i t} \sum_{n=0}^{\infty} P_{n}(\mu)\left(\frac{i D_{n}}{(n+1) r^{n+1}}\right) .
$$

Then notice, using integration by parts and Legendre identities, that

$$
\begin{aligned}
\int_{0}^{\pi} P_{n}(\mu) \cos \theta \sin \theta d \theta & =\frac{2}{3} \delta_{1 n} \text { for } n \geq 0, \\
\int_{0}^{\pi}\left(1-\mu^{2}\right)^{-\frac{1}{2}}\left(\int_{\mu}^{1} P_{n}(x) d x\right) \sin ^{2} \theta d \theta & =\frac{2}{3} \delta_{1 n} \text { for } n \geq 1 .
\end{aligned}
$$

Therefore the force-free condition will only affect the $n=1$ mode. Then as we have the scalings

$$
\frac{\partial u_{r}}{\partial r} \sim O(1), \quad \frac{\partial u_{r}}{\partial \theta} \sim O(1), \quad \frac{\partial u_{\theta}}{\partial r} \sim O\left(\delta^{-1}\right), \quad u_{\theta} \sim O(1), \quad p \sim O(1),
$$

at the two leading orders in $\delta$ only pressure and the viscous stress $\sim \partial u_{\theta} / \partial r$ will contribute to the force, leading to

$$
\boldsymbol{F}=\frac{4 \pi}{3} e^{i t}\left[-\frac{i V_{1}}{2}+\frac{\delta(1+i)\left(W_{1}+V_{1}\right)}{4}+O\left(\delta^{2}\right)\right] \mathbf{e}_{z}
$$

For the spherical body to be force-free we thus need $V_{1}=W_{1}=0$. In other words, if the boundary conditions are such that $V_{1} \neq 0$ and $W_{1} \neq 0$ for a fixed sphere, then the force-free sphere will undergo additional oscillatory motion to compensate and lead to $V_{1}=W_{1}=0$ overall. Physically the $V_{1}$ mode corresponds to the sphere undergoing translational oscillations. So for the body to be force free the translational oscillations are suppressed. At $O(\epsilon)$, since the behaviour is linear, for most angular surface boundary conditions, $W_{1}$ will be directly dependent on the value of $V_{1}$ so a condition restricting translational oscillations would be anticipated to effect the angular motion at this mode too. 
11.2.2 Force-free motion at $O\left(\epsilon^{2}\right)$

In order for the motion of the sphere at $O(\epsilon)$ to be force-free, we saw that two of the surface coefficients become zero. Beyond that, the model has not fundamentally changed. We can thus use our mathematical framework to calculate the velocity of translation at $O\left(\epsilon^{2}\right)$ in terms of the force generated by the oscillating body which was force-free at first order.

We use $\tilde{V}$ to denote the non-dimensional time-averaged velocity of the body at order $\epsilon^{2}$. In order to use the same formulation as above, we move into a frame of reference where the body undergoes no net motion at $O\left(\epsilon^{2}\right)$ in the $\mathrm{z}$ direction. Mathematically, this keeps all the boundary conditions the same as above except now requires in the far field that

$$
\psi \sim-\epsilon^{2} \tilde{V} \frac{r^{2}}{2}\left(1-\mu^{2}\right), \quad r \rightarrow \infty .
$$

As this is an outer boundary condition, the form of the second-order inner solution remains unchanged. Therefore the main change is in the second-order outer solution. The general form of this outer streaming is still given by (61) (which is still a Stokes approximation since the non-linear forcing term can be neglected due to its exponential decay rate). The difference is that applying the new boundary condition (109) allows one more term than before to be non-zero so we have

$$
\begin{array}{r}
\left\langle\psi_{2}^{o}\right\rangle=\tilde{R}_{0}\left(\int_{\mu}^{1} P_{0}(x) d x\right)+\left(-\tilde{V} r^{2}+\tilde{T}_{1} r^{-1}+\tilde{S}_{1} r\right)\left(\int_{\mu}^{1} P_{1}(x) d x\right) \\
+\sum_{n=2}^{\infty}\left(\tilde{T}_{n} r^{-n}+\tilde{S}_{n} r^{-(n-2)}\right)\left(\int_{\mu}^{1} P_{n}(x) d x\right) .
\end{array}
$$

Then when comparing this outer solution to the inner solution (65), (66) and (67) all still hold for $k \neq 1$ but for $k=1$ we instead have

$$
\begin{gathered}
L_{1}=\tilde{T}_{1}+\tilde{S}_{1}-\tilde{V}, \\
\frac{M_{1}}{\delta}=\tilde{S}_{1}-\tilde{T}_{1}-2 \tilde{V} .
\end{gathered}
$$

In order to determine the value of $\tilde{V}$ we enforce that the time-averaged second-order solution be force-free so we have

$$
\boldsymbol{F}=\left\langle\left.\int(\boldsymbol{\sigma} \cdot \boldsymbol{n})\right|_{r=R(\mu, t)} d S\right\rangle=\mathbf{0} .
$$

Contributions to this integral will come from linear terms involving the internal second-order solution, $\psi_{2}^{i}$, as well as non-linear terms involving the first order solution $\psi_{1}$.

As the form of the first-order solution has not changed, the contributions from those non-linear terms remain the same as when we restrict $\tilde{V}=0$ (i.e. no second order translation). However the second-order internal solution will give 
a slightly different contribution as the values of the constants of integration $N_{k}$ and $Q_{k}$ have changed.

Looking at the second-order internal solution only the constants of integration $L_{k}, M_{k}, N_{k}$ and $Q_{k}$ could be different. But changes will only occur for $k=1$ since for other values of $k$ the outer solution is as before. Furthermore, the constants $L_{1}$ and $M_{1}$ are the same as before since their values were determined by the boundary conditions on the surface of the body which are the same. We use the asymptotic matching in order to determine the values of $T_{1}$ and $S_{1}$ in terms of the known values $L_{1}$ and $M_{1}$ and this is then used to calculate the new values of $N_{1}$ and $Q_{1}$.

In this new system we have

$$
\begin{gathered}
L_{1}=\left(\tilde{T}_{1}+\frac{\tilde{V}}{2}\right)+\left(\tilde{S}_{1}-\frac{3 \tilde{V}}{2}\right), \\
\frac{M_{1}}{\delta}=\left(\tilde{S}_{1}-\frac{3 \tilde{V}}{2}\right)-\left(\tilde{T}_{1}+\frac{\tilde{V}}{2},\right), \\
\frac{N_{1}}{\delta^{2}}=\tilde{T}_{1}-\tilde{V}=\left(\tilde{T}_{1}+\frac{\tilde{V}}{2}\right)-\frac{3 \tilde{V}}{2}, \\
\frac{Q_{1}}{\delta^{3}}=-\tilde{T}_{1}=-\left(\tilde{T}_{1}+\frac{\tilde{V}}{2}\right)+\frac{\tilde{V}}{2} .
\end{gathered}
$$

Compared to $S_{1}$ and $T_{1}$ in the first-order force-free case, $T_{1}=\tilde{T}_{1}+\tilde{V} / 2$ and $S_{1}=\tilde{S}_{1}-3 \tilde{V} / 2$. Therefore the drag force on the sphere will be the same as before, $-4 \pi \mu S_{1} e_{z}$ plus an extra contribution from the $-3 \tilde{V} / 2$ extra term in $N_{1}$ and the $\tilde{V} / 2$ term in $Q_{1}$.

Knowing $\hat{\boldsymbol{n}}$ from (101) and that the direction of $F$ is still in the $\boldsymbol{e}_{z}$ direction means we have

$$
\boldsymbol{F}=\left\langle\left.\int\left[\left(\sigma_{r r}-\epsilon \frac{\partial R}{\partial \theta} \sigma_{\theta r}\right) \cos \theta-\left(\sigma_{\theta r}-\epsilon \frac{\partial R}{\partial \theta} \sigma_{\theta \theta}\right) \sin \theta\right]\right|_{r=R(\mu, t)} d S\right\rangle \boldsymbol{e}_{z}
$$

We see that the extra contributions to $F$ can only come from the linear terms evaluated at $r=1$ (so $\eta=0$ ) i.e.

$\sigma_{r r} \cos \theta-\sigma_{\theta r} \sin \theta=\left[\left(-p+\delta^{2} \frac{\partial u_{r}}{\partial r}\right) \cos \theta-\frac{\delta^{2}}{2}\left(\frac{1}{r} \frac{\partial u_{r}}{\partial \theta}+\frac{\partial u_{\theta}}{\partial r}-\frac{u_{\theta}}{r}\right) \sin \theta\right]$.

In $\sigma_{\theta r}$ there will only be an extra $N_{1}$ contribution arising from the $\partial u_{\theta} / \partial r$ term. Any extra contribution from $\sigma_{r r}$ will come from the pressure term. The non-dimensional second-order equation for the pressure is

$$
\epsilon\langle\boldsymbol{u} \cdot \nabla \boldsymbol{u}\rangle=-\nabla\langle\boldsymbol{p}\rangle+\frac{\delta^{2}}{2} \mu \nabla^{2}\langle\boldsymbol{u}\rangle,
$$

where the pressure scales as $p \sim \rho a U \omega$. Then $\langle\boldsymbol{u} \cdot \nabla \boldsymbol{u}\rangle$ gives a contribution in terms of $\psi_{1}$ only. There is however an extra contribution coming from $\nabla^{2}\langle\boldsymbol{u}\rangle$. 
By looking at the $\theta$ component of this equation, and noticing that we are evaluating at $r=1$ in the integral, we see we are only interested in terms with no dependence of $\eta$. Then it can be found that the $N_{k}$ and $Q_{k}$ contribution to $p$ is given by

$$
p=\frac{3}{2} \delta^{2}\left(\frac{Q_{1}}{\delta^{3}}\right) P_{1}(\mu) .
$$

The extra contribution to $F$ coming from the $\partial u_{\theta} / \partial r$ term in $\sigma_{\theta r}$ and from the pressure is

$$
\delta^{2} \int_{0}^{2 \pi} \int_{0}^{\pi}\left[\left(-\frac{3 \tilde{V}}{4}\right) \sin ^{3} \theta-\left(\frac{3 \tilde{V}}{4}\right) \cos ^{2} \theta \sin \theta\right] d \theta d \phi=-3 \pi \delta^{2} V .
$$

Therefore the total non-dimensional force on the spherical body is

$$
\mathbf{F}=\epsilon^{2}\left(-4 \pi S_{1}-3 \pi \delta^{2} \tilde{V}\right) \boldsymbol{e}_{z} .
$$

For the body to be force free its dimensional velocity must therefore be

$$
\tilde{V}=-\frac{4}{3 \delta^{2}}(a \omega) S_{1}
$$

which we can use to calculate a numerical value for $\tilde{V}$.

The relationship between the force on the body and its velocity is given by substituting this value of $S_{1}$ into (99) giving

$$
\boldsymbol{F}=3 \pi \epsilon^{2} \delta^{2} \tilde{V}\left(\rho a^{3} \omega\right) \boldsymbol{e}_{z} .
$$

Then substituting the scaling for $\delta^{2}$ given by (7) finally leads to

$$
\boldsymbol{F}=6 \pi \epsilon^{2} \tilde{V}(a \rho \nu) \boldsymbol{e}_{z} .
$$

We recognise the standard result for a solid sphere translating at speed $\epsilon^{2} \tilde{V}$ in a Stokes flow. Since outside the boundary layer the flow is a Stokes flow, such a similarity was in fact expected (corrections for non-sphericity are expected at higher orders in $\epsilon$ ).

\section{Conclusion}

In this paper we have mathematically derived the steady streaming flow generated by arbitrary axisymmetric shape oscillations of a spherical body. The final solution, and thus the main result of this paper, is quantified in Eqs. (72)-(75).

Our model, which agrees with classical results, shows that a net force is generated in the far field only when two adjacent surface modes are excited. If the body is free to move, this force will cause the body to move with a net velocity, which we derived, given by a balance between that streaming force and the Stokes drag (Eq. 124).

Having kept the boundary forcing arbitrary makes our model applicable to a wide range of microorganisms and microfluidic devices. Future work could 
involve determining the impact of inertia on the optimal swimming shape and associated efficiency of larger microorganisms (such as Spirostomum [43]) which may swim in regimes with non-negligible convective inertia. In addition, using this framework the study of active colloids could be extended to inertial regimes, from the extensively studied low Reynolds number regimes [52].

Recent experimental work has used bubbles embedded in free-moving hollow bodies to generate propulsion [32] and our formalism will be directly applicable to this new class of synthetic swimmers. In particular, in Ref. [41] we used our calculations to determine the streaming flow and force generated by such micron-sized "Armoured Microbubbles". Our analysis could be extended to a wider range of experimental parameters and could help improve future designs.

\section{Acknowledgements}

This work was funded in part by the EPSRC (TS) and the European Union through a Marie Curie CIG Grant (EL).

\section{A Out-of-phase streaming around a bubble}

In order to apply the general steady streaming model (72) specifically to a bubble the boundary condition of tangential stress on the boundary of the spherical body,

$$
\boldsymbol{n} \cdot \boldsymbol{\sigma} \cdot \boldsymbol{t}=0 \quad \text { at } \quad r=R, \theta=\Theta
$$

needs to be applied. This will determine the angular motion on the surface of the bubble $W_{n}$ and $F_{n}$ in terms of a prescribed radial motion $V_{n}$.

\section{A.1 Boundary condition}

We denote the unit tangent vector to the body's surface in the plane through the axis of axisymmetry $\boldsymbol{t}$ and the normal vector $\boldsymbol{n}$. Both can be calculated in terms of $\mathbf{e}_{z}$ and $\mathbf{e}_{\theta}$ measured from the center of the rest position of the body giving

$$
\begin{gathered}
\boldsymbol{t}=\mathbf{e}_{\theta}+\frac{\partial R}{\partial \theta} \mathbf{e}_{r}+O\left(\epsilon^{3}\right), \\
\boldsymbol{n}=\mathbf{e}_{r}-\frac{\partial R}{\partial \theta} \mathbf{e}_{\theta}+O\left(\epsilon^{3}\right) .
\end{gathered}
$$

Using these equations the no stress condition can be expanded in terms of $\epsilon$ giving the first two terms as

$$
\sigma_{\theta r}+\frac{\partial R}{\partial \theta_{0}}\left(\sigma_{r r}-\sigma_{\theta \theta}\right)+O\left(\epsilon^{3}\right)=0 \quad \text { at } \quad r=R, \theta=\Theta,
$$

becoming

$$
\sigma_{\theta r}+(R-1) \frac{\partial \sigma_{\theta r}}{\partial r}+\left(\Theta-\theta_{0}\right) \frac{\partial \sigma_{\theta r}}{\partial \theta}+\left(\sigma_{r r}-\sigma_{\theta \theta}\right) \frac{\partial R}{\partial \theta}+O\left(\epsilon^{3}\right)=0 \quad \text { at } \quad r=1, \theta=\theta_{0}
$$


A.2 Leading-order solution

At $O(\epsilon)$, Eq. 131 reduces to the non-dimensional equation

$$
\left(\frac{1}{r} \frac{\partial u_{r}}{\partial \theta}+\frac{\partial u_{\theta}}{\partial r}-\frac{u_{\theta}}{r}\right)=0 \quad \text { at } \quad r=1, \theta=\theta_{0} .
$$

By substituting in the known first-order solution $\psi_{1},(26)$, this equation can be used to determine $W_{n}$ giving

$$
W_{n}=-n V_{n}+\delta\left[\frac{2 n(n+2)}{(1+i)} V_{n}\right]+\delta^{2}\left[i n(n+2)^{2} V_{n}\right]+O\left(\delta^{3}\right) .
$$

\section{A.3 Second-order solution}

At $O\left(\epsilon^{2}\right)$, after Taylor expansion of $\sigma_{\theta r}$ (131) reduces to the non-dimensional equation

$$
\sigma_{\theta r}^{\left(\epsilon^{2}\right)}=-(R-1) \frac{\partial \sigma_{\theta r}^{(\epsilon)}}{\partial R}-\left(\Theta-\theta_{0}\right) \frac{\partial \sigma_{\theta r}^{(\epsilon)}}{\partial \theta}-\frac{\partial R}{\partial \theta_{0}}\left(\sigma_{r r}^{(\epsilon)}-\sigma_{\theta \theta}^{(\epsilon)}\right) \quad \text { at } \quad r=R, \theta=\Theta,
$$

where superscripts indicate the order at which each term is to be taken. Upon substitution of $R, \Theta$ and $\sigma$,(134) becomes

$$
\begin{gathered}
\left(\left(1-\mu^{2}\right) \frac{\partial^{2} \psi_{2}^{i}}{\partial \mu^{2}}+2 \frac{\partial \psi_{2}^{i}}{\partial r}-\frac{\partial^{2} \psi_{2}^{i}}{\partial r^{2}}\right)= \\
\Re\left[\frac{i}{\omega} \sum_{n=0}^{\infty} V_{n} P_{n}(\mu) e^{i t}\right] \Re\left[\left(\left(1-\mu^{2}\right) \frac{\partial^{3} \psi_{1}}{\partial \mu^{2} \partial r}+3 \frac{\partial^{2} \psi_{1}}{\partial r^{2}}-\frac{\partial^{3} \psi_{1}}{\partial r^{3}}-3\left(1-\mu^{2}\right) \frac{\partial^{2} \psi_{1}}{\partial \mu^{2}}-4 \frac{\partial \psi_{1}}{\partial r}\right)\right] \\
+\Re\left[\frac{\epsilon i}{\omega} \sum_{n=1}^{\infty} W_{n}\left(\int_{\mu}^{1} P_{n}(x) d x\right) e^{i t}\right] \Re\left[\frac{\mu}{\left(1-\mu^{2}\right)}\left(\left(1-\mu^{2}\right) \frac{\partial^{2} \psi_{1}}{\partial \mu^{2}}+2 \frac{\partial \psi_{1}}{\partial r}-\frac{\partial^{2} \psi_{1}}{\partial r^{2}}\right)\right. \\
\left.+\left(\frac{\partial\left(\left(1-\mu^{2}\right) \frac{\partial^{2} \psi_{1}}{\partial \mu^{2}}\right)}{\partial \mu}+2 \frac{\partial^{2} \psi_{1}}{\partial r \partial \mu}-\frac{\partial^{3} \psi_{1}}{\partial r^{2} \partial \mu}\right)\right]-\Re\left[\frac{\epsilon i}{\omega} \sum_{n=0}^{\infty} n(n+1) V_{n}\left(\int_{\mu}^{1} P_{n}(x) d x\right) e^{i t}\right] \\
\times\left[2\left(3 \frac{\partial \psi_{1}}{\partial \mu}-2 \frac{\partial \psi_{1}}{\partial r \partial \mu}-\frac{\mu}{\left(1-\mu^{2}\right)} \frac{\partial \psi_{1}}{\partial r}\right)\right] \quad \text { at } \quad r=1, \theta=\theta_{0} . \quad \text { (135) }
\end{gathered}
$$

The forcing on the right-hand side of (135) can be calculated explicitly from the derivatives of $\psi_{1},(26)$, simplifying the equation to

$$
\begin{gathered}
\left\langle\left(1-\mu^{2}\right) \frac{\partial^{2} \psi_{2}^{i}}{\partial \mu^{2}}+2 \frac{\partial \psi_{2}^{i}}{\partial r}-\frac{\partial^{2} \psi_{2}^{i}}{\partial r^{2}}\right\rangle=\frac{i}{2} \sum_{k=0}^{\infty} \sum_{n=0}^{\infty} \sum_{m=1}^{\infty} a_{k n m}\left\{-m\left(m^{2}+2\right) V_{m} \bar{V}_{n}\right. \\
+(m+2)^{2} W_{m} \bar{V}_{n}-4 m(m+1) \bar{W}_{n} V_{m}+\bar{V}_{n}\left(W_{m}+m V_{m}\right)\left[\frac{2 i}{\delta^{2}}+\frac{(m+3)(1+i)}{\delta}+\right. \\
\left.\frac{m(2 m+3)}{2}\right]-W_{m}\left(\bar{W}_{n}+n \bar{V}_{n}\right) \frac{(1-i)}{\delta}+W_{m} \bar{V}_{n} n(n+2)-(n+2) \bar{W}_{n} W_{m} \\
+\sum_{j=1}^{\infty}\left(\frac{C_{n j}}{j(j+1)}\right)\left[\frac{1}{\delta} \frac{W_{m}\left(\bar{W}_{j}+j \bar{V}_{j}\right)(1-i)}{j(j+1)}+\frac{(j+2) W_{m}\left(\bar{W}_{j}-j \bar{V}_{j}\right)}{j(j+1)}\right. \\
\left.\left.+2 m(m+1) V_{m} \bar{W}_{j}\right]\right\} \int_{\mu}^{1} P_{k}(x) d x
\end{gathered}
$$


The left hand side of (135) can be evaluated using $\psi_{2}^{i}$ from (56) and the value of the three constants of integration, $L_{k}, M_{k}$ and $N_{k}$, are needed. $L_{k}$ and $M_{k}$ were calculated in (57) and (58). From the asymptotic matching $L_{k}$ and $M_{k}$ are known in terms of $T_{k}$ and $S_{k},(65)$ and (66). Matching at one higher order determines $N_{k}$ in terms of $T_{k}$ and $S_{k}$, then using (65) and (66), $N_{k}$ can be written in terms of $L_{k}$ and $M_{k}$ giving

$$
N_{k}=\left[\frac{(2-k) k}{2} L_{k}+\frac{(1-2 k)}{2}\left(\frac{M_{k}}{\delta}\right)\right] \delta^{2}
$$

Equation (135) leads to an equality for $\sum_{k=1}^{\infty} F_{k}\left(\int_{\mu}^{1} P_{k}(x) d x\right)$ at leading order, $O(1)$. As this must hold for all $\mu \in[-1,1]$, the coefficients of $\left(\int_{\mu}^{1} P_{k}(x) d x\right)$ must equate for each $k$, and thus this will give an equation for every $F_{k}$ separately. Upon substitution of (133) the equation for $F_{k}$ reduces to

$$
\begin{gathered}
\frac{F_{k}^{(\delta)}}{\delta}=-\sum_{n=0}^{\infty} \sum_{m=1}^{\infty} a_{k n m}\left[m(m+4+n) \frac{i}{2} \bar{V}_{n} V_{m}+\sum_{j=1}^{\infty}\left(\frac{C_{n j}}{(j+1)}\right)\left(\frac{i}{2} m \bar{V}_{m} V_{j}\right)\right] \\
-\frac{3 k}{(2 k+1)} V_{0} \bar{V}_{k} i-\sum_{n=0}^{\infty} \sum_{m=1}^{\infty} \frac{9 k g_{k n m}}{2(2 k+1)} V_{m} \bar{V}_{n} i+\sum_{n=1}^{\infty} \sum_{m=1}^{\infty} \frac{3 k f_{k n m}}{2(2 k+1)} \frac{(n+2) i \bar{V}_{n} V_{m}}{(n+1)(m+1)} \\
+\sum_{n=0}^{\infty} \sum_{m=1}^{\infty} \frac{a_{k n m}}{(2 k+1)}\left[m\left(n^{2}-4 n m-4 n+m^{2}-m-3\right) i \bar{V}_{n} V_{m}\right. \\
\left.+\sum_{j=1}^{\infty}\left(\frac{C_{n j}}{(j+1)}\right) m(3 m+5) i V_{m} \bar{V}_{j}\right] .
\end{gathered}
$$

Then substituting $F_{k}^{(\delta)}$ into (74) and (75) finally gives

$$
\begin{aligned}
T_{k}=\Re\left\{\frac{\left(1-k^{2}\right)}{(2 k+1)} i V_{0} \bar{V}_{k}+\sum_{n=1}^{\infty} \sum_{m=1}^{\infty} \frac{\left(k^{2}-k-1\right)(n+2)}{2(2 k+1)(n+1)(m+1)} f_{k n m} i \bar{V}_{n} V_{m}\right. \\
+\sum_{n=0}^{\infty} \sum_{m=1}^{\infty} \frac{3\left(1-k^{2}\right)}{2(2 k+1)} i V_{m} \bar{V}_{n} g_{k n m}+\sum_{n=0}^{\infty} \sum_{m=1}^{\infty} \frac{a_{k n m}}{2(2 k+1)}\left[m \left(n^{2}-4 n m-4 n\right.\right. \\
\left.\left.\left.+m^{2}-m-3\right) i \bar{V}_{n} V_{m}+\sum_{j=1}^{\infty}\left(\frac{C_{n j}}{j+1}\right) m(3 m+5) i V_{m} \bar{V}_{j}\right]\right\}+O(\delta)
\end{aligned}
$$

and

$$
\begin{gathered}
S_{k}=\Re\left\{\frac{k(k+2)}{(2 k+1)} i V_{0} \bar{V}_{k}-\sum_{n=1}^{\infty} \sum_{m=1}^{\infty} \frac{(2 k+4) k(n+2)}{4(2 k+1)(n+1)(m+1)} f_{k n m} i \bar{V}_{n} V_{m}\right. \\
+\sum_{n=0}^{\infty} \sum_{m=1}^{\infty} \frac{3 k(k+2)}{2(2 k+1)} i V_{m} \bar{V}_{n} g_{k n m}-\sum_{n=0}^{\infty} \sum_{m=1}^{\infty} \frac{a_{k n m}}{2(2 k+1)}\left[m \left(n^{2}-4 n m-4 n\right.\right. \\
\left.\left.\left.+m^{2}-m-3\right) i \bar{V}_{n} V_{m}+\sum_{j=1}^{\infty}\left(\frac{C_{n j}}{j+1}\right) m(3 m+5) i V_{m} \bar{V}_{j}\right]\right\}+O(\delta)
\end{gathered}
$$

which with (73) give the value of all the constants in the streaming solution (72). 


\section{B In-phase streaming around a bubble}

Notice, from the solution of the steady streaming around a bubble given in Appendix A, that all terms in (140), (139) and (73) are multiplies of $i V_{m} \bar{V}_{k}$ for some integers $m, k \geq 0$. As such Appendix A only gives the solution for out-of-phase motion of the bubble, as otherwise that solution is identically zero. Therefore for these cases, the steady streaming needs to be calculated to the next order, namely $O(\delta)$.

Since here the analysis is to one higher order in $\delta$ this could require more stringent conditions than $\epsilon \ll \delta \ll 1$ relationship. However the order change is due to terms being identically zero which we expect to continue at higher orders in $\epsilon$ so the same relationship should hold.

\section{B.1 Inner-solution at third order in $\delta$}

In order to find the steady streaming at $O(\delta)$, the asymptotic matching must be carried out at one higher order. As such the inner second-order solution must be calculated to an extra order in $\delta$. Therefore more terms will be required in the $\delta$ expansions, so we first return to the second-order, inner governing equation

$$
\frac{\delta^{2}}{2}\left\langle D^{4} \psi_{2}^{i}\right\rangle=\frac{1}{r^{2}}\left\langle\frac{\partial\left(\psi_{1}, D^{2} \psi_{1}\right)}{\partial(r, \mu)}+2 L \psi_{1} D \psi_{1}\right\rangle .
$$

When expanding the left hand side in $\delta$, the $O(1)$ terms in $\delta$ now contribute to the streaming as well as the $O\left(\delta^{-2}\right)$ term so we have

$$
\frac{\delta^{2}}{2}\left\langle D^{4} \psi_{2}^{i}\right\rangle=\frac{1}{2 \delta^{2}} \frac{\partial^{4}\left\langle\psi_{2}^{i}\right\rangle}{\partial \eta^{4}}+\left(1-\mu^{2}\right) \frac{\partial^{4}\left\langle\psi_{2}^{i}\right\rangle}{\partial \eta^{2} \partial \mu^{2}}+O(\delta) .
$$

From (56), the $O(1)$ (leading-order) solution of $\left\langle\psi_{2}^{i}\right\rangle$ is known. The second term in (142) will only make an $O(1)$ or lower contribution from this term so the governing equation for the first three orders of $\left\langle\psi_{2}^{i}\right\rangle$ can be simplified to

$$
\begin{aligned}
& \frac{\partial^{4}\left\langle\psi_{2}^{i}\right\rangle}{\partial \eta^{4}}=\frac{2 \delta^{2}}{r^{2}}\left\langle\frac{\partial\left(\psi_{1}, D^{2} \psi_{1}\right)}{\partial(r, \mu)}+2 L \psi_{1} D \psi_{1}\right\rangle \\
& +\delta^{2} \sum_{k=1}^{\infty} \sum_{n=0}^{\infty} \sum_{m=1}^{\infty} a_{k n m}\left[2 k(k+1)\left(W_{m}+m V_{m}\right) \bar{V}_{n} e^{-(1+i) \eta}\right]\left(\int_{\mu}^{1} P_{k}(x) d x\right)+O\left(\delta^{3}\right)
\end{aligned}
$$

Expanding the term $\left\langle\partial\left(\psi_{1}, D^{2} \psi_{1}\right) / \partial(r, \mu)+2 L \psi_{1} D \psi_{1}\right\rangle$ to $O\left(\delta^{2}\right)$ and substituting into (143) then gives an equation for $\left\langle\partial^{4} \psi_{2}^{i} / \partial \eta^{4}\right\rangle$ which can be integrated twice to find that the $O\left(\delta^{2}\right)$ (only) term in $\left\langle\partial^{2} \psi_{2}^{i} / \partial \eta^{2}\right\rangle$ is

$$
\begin{array}{r}
\left\langle\frac{\partial^{2} \psi_{2}^{i}}{\partial \eta^{2}}\right\rangle^{\left(\delta^{2}\right)}=\sum_{k=1}^{\infty}\left(\int_{\mu}^{1} P_{k}(x) d x\right) \sum_{n=0}^{\infty} \sum_{m=1}^{\infty} a_{k n m}\left\{-m\left(\bar{W}_{n}+n \bar{V}_{n}\right) V_{m} i\left(\frac{n}{2}-m-3\right)\right. \\
+\left(\bar{W}_{n}+n \bar{V}_{n}\right)\left(W_{m}+m V_{m}\right)\left(\frac{i}{2}(1-m+n)-\left(2+\frac{n}{2}\right)\right) \\
+\left(W_{m}+m V_{m}\right) \bar{V}_{n} i\left(-\frac{5 n^{2}}{4}-\frac{21 n}{4}+n m+m+\frac{m^{2}}{4}-3-t(t+1)\right) \\
+\sum_{j=1}^{\infty}\left(\frac{C_{n j}}{j(j+1)}\right)\left[\left(\bar{W}_{j}+j \bar{V}_{j}\right)\left(W_{m}+m V_{m}\right)\left((1+i)-\frac{1}{2}(m+i j)+j\right)\right. \\
\left.\left.+\bar{V}_{j}\left(W_{m}+m V_{m}\right) i j(2 j+6-m)\right]\right\}
\end{array}
$$


The $O\left(\delta^{2}\right)$ contribution to $\left\langle\psi_{2}^{i}\right\rangle$ can be calculated by integrating twice more but in order to satisfy the no tangential stress boundary condition only $\left\langle\partial^{2} \psi_{2}^{i} / \partial \eta^{2}\right\rangle$ is required at $O\left(\delta^{2}\right)$.

Notice that every term in $\left\langle\psi_{2}^{i}\right\rangle$ up to and including $O(1)$ is a multiple of $W_{n}+n V_{n}$ for some $n$ so every term will drop an order when the first order stress condition $W_{n}=-n V_{n}+$ $O(\delta)$ is applied. Similarly, higher-order terms in $\left\langle\psi_{2}^{i}\right\rangle$ will also be multiples of $W_{n}+n V_{n}$ since in $\left\langle\partial\left(\psi_{1}, D^{2} \psi_{1}\right) / \partial(r, \mu)+2 L \psi_{1} D \psi_{1}\right\rangle$ every term is a multiple of $B_{n} \propto\left(W_{n}+n V_{n}\right)$ and in $\left\langle D^{4} \psi_{2}^{i}\right\rangle$ extra terms in its delta expansion will be in terms of lower orders of $\left\langle\psi_{2}^{i}\right\rangle$ which are also proportional to $W_{n}+n V_{n}$. Therefore even when calculating the streaming to $O(\delta)$ for a bubble, only the first three terms up to $O(1)$ are needed in the equation for the inner streaming.

\section{B.2 Constants of integration}

The second order inner solution $\left\langle\psi_{2}^{i}\right\rangle$ is of the form

$$
\left\langle\psi_{2}^{i}\right\rangle=\sum_{k=1}^{\infty}\left(\int_{\mu}^{1} P_{k}(x) d x\right)\left[f_{k}(\eta)+g_{k}(\eta) \delta+h_{k}(\eta) \delta^{2}+L_{k}+M_{k} \eta+N_{k} \eta^{2}+O\left(\delta^{3}\right)\right]
$$

where $f$ and $g$ are known from (56) and $h$ could be found by integrating (144). The notangential stress boundary condition then gives

$$
\begin{aligned}
& \left\langle\left(1-\mu^{2}\right) \frac{\partial^{2} \psi_{2}^{i}}{\partial \mu^{2}}+2 \frac{\partial \psi_{2}^{i}}{\partial r}-\frac{\partial^{2} \psi_{2}^{i}}{\partial r^{2}}\right\rangle=\sum_{k=1}^{\infty}\left\{\frac{1}{\delta^{2}}\left(-\frac{\partial^{2} f_{k}}{\partial \eta^{2}}\right)+\frac{1}{\delta}\left(2 \frac{\partial f_{k}}{\partial \eta}-\frac{\partial^{2} g_{k}}{\partial \eta^{2}}\right)\right. \\
& \left.+\left[-k(k+1) f_{k}(\eta)+2 \frac{\partial g_{k}}{\partial \eta}-\frac{\partial^{2} h_{k}}{\partial \eta^{2}}+2 \frac{M_{k}}{\delta}-2 \frac{N_{k}}{\delta^{2}}-k(k+1) L_{k}\right]\right\}\left(\int_{\mu}^{1} P_{k}(x) d x\right)
\end{aligned}
$$

The $O\left(\delta^{-2}\right)$ terms will cancel with the $O\left(\delta^{-2}\right)$ quantity in (136). The $O\left(\delta^{-1}\right)$ terms do not cancel exactly but when taking $W_{n}=-n V_{n}+O(\delta)$ (the first order bubble condition) they do. Therefore the $O(1)$ terms will give the leading-order behaviour for which the value of the constants $L_{k}, M_{k}$ and $N_{k}$ are needed. The constants $L_{k}$ and $M_{k}$ were calculated in (57) and (58) and $N_{k}$ can be written in terms of $L_{k}$ and $M_{k},(137)$.

Equation (146) can then be equated with (136) to give the algebraic condition for no tangential stress. This will give a condition on $M_{k}$ and $L_{k}$ but $L_{k}$ is uniquely determined by the boundary condition: the radial velocity of the bubble equals the radial velocity of the fluid adjacent to the bubble. $M_{k}$ was also determined but is a function of the unknown $F_{k}$ which this no tangential stress condition will determine. However $F_{k}$ uniquely determines 
$M_{k}$ so this equation can be considered as just determining $M_{k}$ giving

$$
\begin{gathered}
-(2 k+1)\left(\frac{M_{k}}{\delta}\right)=(-3 k) L_{k}+\sum_{n=0}^{\infty} \sum_{m=1}^{\infty} a_{k n m}\left(\frac { 1 } { \delta } \left\{\left(W_{m}+m V_{m}\right)\left(\bar{W}_{n}+n \bar{V}_{n}\right) \frac{(i-1)}{2}\right.\right. \\
+\left(W_{m}+m V_{m}\right) \frac{\bar{V}_{n}}{2}(1-i)(2 n+3)+\sum_{j=1}^{\infty}\left(\frac{C_{n j}}{j(j+1)}\right)\left[\left(\bar{W}_{j}+j \bar{V}_{j}\right)\left(W_{m}+m V_{m}\right) \frac{(1+i)}{2}\right. \\
\left.\left.-\left(W_{m}+m V_{m}\right) \bar{V}_{j} j(1-i)-\frac{W_{m}\left(\bar{W}_{j}+j \bar{V}_{j}\right)(1+i)}{2 j(j+1)}\right]\right\}+\left\{m\left(\bar{W}_{n}+n \bar{V}_{n}\right) V_{m} i\left(\frac{n}{2}-m-2\right)\right. \\
-\left(\bar{W}_{n}+n \bar{V}_{n}\right)\left(W_{m}+m V_{m}\right)\left[\frac{i}{2}(n-m)+\left(\frac{n}{2}-\frac{1}{2}\right)\right]-\left(W_{m}+m V_{m}\right) \bar{V}_{n} i\left[-\frac{5 n^{2}}{4}-\frac{9 n}{4}+n m\right. \\
\left.+\frac{m^{2}}{4}+1-\frac{3}{2} k(k+1)+\frac{\left(2 m^{2}+3 m\right)}{4}\right]-\frac{i}{2} W_{m} \bar{V}_{n} n(n+2)+\frac{i}{2}(n+2) \bar{W}_{n} W_{m} \\
+\frac{i}{2} m\left(m^{2}+2\right) V_{m} \bar{V}_{n}-\frac{i}{2}\left[(m+2)^{2} W_{m} \bar{V}_{n}-4 m(m+1) \bar{W}_{n} V_{m}\right] \\
+\sum_{j=1}^{\infty}\left(\frac{C_{n j}}{j(j+1)}\right)\left[-\left(\bar{W}_{j}+j \bar{V}_{j}\right)\left(W_{m}+m V_{m}\right)\left(\frac{(1+i)}{2}-\frac{1}{2}(m+i j)+j\right)\right. \\
\left.\left.\left.-\bar{V}_{j}\left(W_{m}+m V_{m}\right) i j(2 j+4-m)-i m(m+1) V_{m} \bar{W}_{j}-\frac{i}{2}\left(\frac{(j+2) W_{m}\left(\bar{W}_{j}-j \bar{V}_{j}\right)}{j(j+1)}\right)\right]\right\}\right)+O(\delta) .
\end{gathered}
$$

When applying the first-order stress boundary condition (133) all terms drop by one order in $\delta$. Then assuming the $O(1)$ terms cancel (which is required for the result in Appendix A not to give the solution) this gives $M_{k}$ at $O(\delta)$. Then $L_{k}$ can be calculated at $O(\delta)$ by applying (32) at $O(\delta)$ to $\left\langle\psi_{2}^{i}\right\rangle(56)$. This gives

$$
\begin{gathered}
L_{k}=\delta\left\{\sum_{n=0}^{\infty} \sum_{m=1}^{\infty} a_{k n m} \frac{m(m+2)}{2}\left[(1+i) V_{m} \bar{V}_{n}\right]-\sum_{n=0}^{\infty} \sum_{m=0}^{\infty} \frac{m(m+2)}{2}\left[(1+i) V_{m} \bar{V}_{n}\right] g_{k n m}\right. \\
\left.+\sum_{n=1}^{\infty} \sum_{m=1}^{\infty} \frac{(n+2)(m+2) f_{k n m}}{2(n+1)(m+1)}\left[(1+i) \bar{V}_{n} V_{m}\right]-\sum_{m=1}^{\infty} \sum_{n=1}^{\infty} \frac{(n+2)}{2(n+1)(m+1)} f_{k n m}\left[(1-i) \bar{V}_{n} V_{m}\right]\right\},
\end{gathered}
$$

and the simplified $M_{k}$ expression

$$
\begin{aligned}
& \left(\frac{M_{k}}{\delta}\right)=\frac{(3 k)}{(2 k+1)} L_{k}-\delta \sum_{n=0}^{\infty} \sum_{m=1}^{\infty} \frac{a_{k n m}}{(2 k+1)}\left\{-n m(n+2)(2 m+1)(1-i) V_{m} \bar{V}_{n}\right. \\
& \quad+m(m+2)\left[\frac{n^{2}}{4}+\frac{9 n}{4}-\frac{5 m^{2}}{4}+\frac{3}{2} t(t+1)-\frac{5 m}{4}\right](1+i) V_{m} \bar{V}_{n} \\
& \left.+\sum_{j=1}^{\infty}\left(\frac{C_{n j}}{j(j+1)}\right)\left[m j(m+1)(j+2)(1-i) V_{m} \bar{V}_{j}-(j+4) m j(m+2)(1+i) V_{m} \bar{V}_{j}\right]\right\}
\end{aligned}
$$




\section{B.3 Outer streaming constants}

Using the matching conditions (68) and (69) we finally obtain

$$
\begin{gathered}
T_{k}=\delta \Re\left(\sum_{n=1}^{\infty} \sum_{m=1}^{\infty} \frac{\left(1-k^{2}\right)(n+2)}{2(2 k+1)(n+1)(m+1)} f_{k n m}\left[(m+2)(1+i) \bar{V}_{n} V_{m}-(1-i) \bar{V}_{n} V_{m}\right]\right. \\
-\sum_{n=0}^{\infty} \sum_{m=0}^{\infty} \frac{\left(1-k^{2}\right) m(m+2)}{2(2 k+1)}\left[(1+i) V_{m} \bar{V}_{n}\right] g_{k n m}+\sum_{n=0}^{\infty} \sum_{m=1}^{\infty} a_{k n m}\left\{m ( m + 2 ) \left(\frac{n^{2}}{4}+\frac{9 n}{4}\right.\right. \\
\left.-\frac{5 m^{2}}{4}-\frac{5 m}{4}+1+\frac{1}{2} k^{2}+\frac{3}{2} k\right) \frac{(1+i)}{2(2 k+1)} V_{m} \bar{V}_{n}-n m(n+2)(2 m+1) \frac{(1-i)}{2(2 k+1)} V_{m} \bar{V}_{n} \\
\left.\left.+\sum_{j=1}^{\infty}\left(\frac{C_{n j}}{(j+1)}\right)\left[m(m+1)(j+2) \frac{(1-i)}{2(2 k+1)} V_{m} \bar{V}_{j}-(j+4) m(m+2) \frac{(1+i)}{2(2 k+1)} V_{m} \bar{V}_{j}\right]\right\}\right),
\end{gathered}
$$

and

$$
\begin{gathered}
S_{k}=\delta \Re\left(\sum_{n=1}^{\infty} \sum_{m=1}^{\infty} \frac{k(k+2)(n+2)}{2(n+1)(m+1)(2 k+1)} f_{k n m}\left[(m+2)(1+i) \bar{V}_{n} V_{m}-(1-i) \bar{V}_{n} V_{m}\right]\right. \\
-\sum_{n=0}^{\infty} \sum_{m=0}^{\infty} \frac{k(k+2) m(m+2)}{2(2 k+1)}\left[(1+i) V_{m} \bar{V}_{n}\right] g_{k n m}+\sum_{n=0}^{\infty} \sum_{m=1}^{\infty} a_{k n m}\left\{-m(m+2)\left[\frac{n^{2}}{4}+\frac{9 n}{4}\right.\right. \\
\left.-\frac{5 m^{2}}{4}-\frac{5 m}{4}+\frac{1}{2} k(k-1)\right] \frac{(1+i)}{2(2 k+1)} V_{m} \bar{V}_{n}+n m(n+2)(2 m+1) \frac{(1-i)}{2(2 k+1)} V_{m} \bar{V}_{n} \\
\left.\left.+\sum_{j=1}^{\infty}\left(\frac{C_{n j}}{(j+1)}\right)\left[-m(m+1)(j+2) \frac{(1-i)}{2(2 k+1)} V_{m} \bar{V}_{j}+(j+4) m(m+2) \frac{(1+i)}{2(2 k+1)} V_{m} \bar{V}_{j}\right]\right\}\right),
\end{gathered}
$$

which, with $Y_{k n m}=0$, give the value of all the constants in the Lagrangian streaming solution (72).

\section{References}

1. T. M. Squires and S. R. Quake. Microfluidics: Fluid physics at the nanoliter scale. Rev. Mod. Phys., 77:977-1026, 2005.

2. S. K. Sia and G. M. Whitesides. Microfluidic devices fabricated in poly(dimethylsiloxane) for biological studies. Electrophoresis, 24:3563-3576, 2003.

3. H. A. Stone and S. Kim. Microfluidics: Basic Issues, Applications, and Challenges. AIChE Journal, 47:1250-1254, 2001.

4. H. H. Wensink, J. Dunkel, S. Heidenreich, K. Drescher, R. E. Goldstein, H. Lowen, and J. M. Yeomans. Meso-scale turbulence in living fluids. Proc. Natl. Acad. Sci. USA, 109:14308-14313, 2012.

5. E. Lauga and T. R. Powers. The hydrodynamics of swimming micro-organisms. Rep. Prog. Phys., 72:096601, 2009.

6. K. Drescher, R. E. Goldstein, N. Michael, M. Polin, and I. Tuval. Direct Measurement of the Flow Field around Swimming Microorganisms. Phys. Rev. Lett., 105, 2010.

7. T. J. Pedley and J. O. Kessler. Hydrodynamic phenomena in suspension of swimming microorganisms. Annu. Rev. Fluid Mech., 24:313-358, 1992.

8. D. Saintillan and M. J. Shelley. Instabilities and pattern formation in active particle suspensions: Kinetic theory and continuum simulations. Phys. Rev. Lett., 100:178103, 2008. 
9. J. Dunkel, S. Heidenreich, K. Drescher and H. H. Wensink, M. Bar, and R. E. Goldstein Fluid Dynamics of Bacterial Turbulence. Phys. Rev. Lett., 110:228102, 2013.

10. K. C. Leptos, J. S. Guasto, J. P. Gollub, A. I. Pesci, and R. E. Goldstein. Dynamics of Enhanced Tracer Diffusion in Suspension of Swimming Eukaryotic Microorganisms. Phys. Rev. Lett., 103, 2009.

11. Z. Lin, J. Thiffeault, and S. Childress. Stirring by squirmers. J. Fluid Mech., 669:167$177,2011$.

12. I. M. Zaid, J. Dunkel, and J. M. Yeomans. Levy fluctuations and mixing in dilute suspensions of algae and bacteria. J. R. Soc. Interface, 8:1314-1331, 2011.

13. J. R. Blake. A spherical envelope approach to ciliary propulsion. J. Fluid Mech. 46:199-208, 1971.

14. C. Brennen. An oscillating-boundary-layer theory for cilliary propulsion. J. Fluid Mech., 65:799-824, 1974

15. M. J. Lighthill. On the squirming motion of nearly spherical deformable bodies through liquids at very small Reynolds numbers. Commun. Pur. Appl. Math., 5:109-118, 1952.

16. O. Pak and E. Lauga. Generalized squirming motion of a sphere. J. Eng. Math., 88:1-28, 2014.

17. G. Li and A. M. Ardekani. Hydrodynamic interaction of microswimmers near a wall. PhysRevE, 90, 2014

18. S. E. Spagnolie and E. Lauga. Hydrodynamics of self-propulsion near a boundary: Predictions and accuracy of far-field approximations. J. Fluid Mech., 700:105-147, 2012.

19. K. Ishimoto and E. A. Gaffney. Swimming efficiency of spherical squirmer: Beyond the Lighthill theory. Phys. Rev. E, 90, 2014.

20. S. Wang and A. M. Ardekani. Unsteady swimming of small organisms. J. Fluid Mech., 702:286-297, 2012.

21. K. Ishimoto. A spherical squirming swimmer in unsteady Stokes flow. J. Fluid Mech., 723:163-189, 2013

22. P. M. Rao. Mathematical model for unsteady ciliary propulsion. Math. Comput. Model. $10: 839-851,1988$

23. S. Wang and A. Ardekani. Inertial Squirmer. Phys. Fluids, 24, 2012.

24. A. S. Khair and N. G. Chisholm. Expansions at small Reynolds numbers for the locomotion of a spherical squirmer. Phys. Fluids, 26:011902, 2014.

25. Volker Hessel, Holger Lowe, and Freidhelm Schonfeld. Micromixers - a review on passive and active mixing principles. Chemical Engineerin Science, 8-9:2479-2501, 2005.

26. Nam-Trung Nguyen and Zhigang Wu. Micromixers - a review. J. Micromech. Microeng, $15,2005$.

27. N. Riley. Steady Streaming. Annu. Rev. Fluid Mech., 33:43-65, 2001.

28. Y. Chen and S. Lee. Manipulation of Biological Objects Using Acoustic Bubbles: A Review. Integr. Comp. Biol., pages 1-10, 2014.

29. S. A. Elder. Cavitation Microstreaming. J. Acoust. Soc. Am., 31, 1958.

30. P. Marmottant and S. Hilgenfeldt. A bubble-driven microfluidic transport element for bioengineering. Proc. Natl. Acad. Sci. USA, 101:9523-9527, 2004.

31. P. Marmottant and S. Hilgenfeldt. Controlled vesicle deformation and lysis by single oscillating bubbles. Nature, 423, 2003.

32. J. Feng, J. Yuan, and S. K. Cho. Micropropulsion by an acoustic bubble for navigating microfluidic spaces. Lab. Chip, 15:1554-1562, 2015.

33. C. Wang, B. Rallabandi Hilgenfeldt, and S. Frequency dependence and frequency control of microbubble streaming flows. Phys. Fluids, 25, 2013.

34. D. Ahmed, X. Mao, B. K. Juluri, and T. Huang. A fast microfluidic mixer based on acoustically driven sidewall-trapped microbubbles. Microfluid. Nanofluid., 7:727-731, 2009 .

35. S. S. Wang, Z. J. Jiao, X. Y. Huang, C. Yang, and N. T. Nguyen. Acoustically induced bubbles in a microfluidic channel for mixing enhancement. Microfluid. Nanofluid., 6:847-852, 2009.

36. N. Riley. On a sphere oscillating in a viscous fluid. Q. J. Mech. Appl. Math., 19, 1966.

37. B. J. Davidson and N. Riley. Cavitation Microstreaming. J. Sound Vib., 15:217-233, 1971. 
38. M. S. Longuet-Higgins. Viscous streaming from an oscillating spherical bubble. Proc. Roy. Soc. Lond. A, 454:725-742, 1998.

39. A. O. Maksimov. Viscous streaming from surface waves on the wall of acousticallydriven gas bubbles. Eur. J. Mech. B-Fluid, 26:28-42, 2007.

40. D. Ahmed, M. Lu, A. Nourhani, P.E. Lammert, Z. Stratton, H.S. Muddana, V.H.Crespi, and T.J.Huang. Selectively manipulable acoustic-powered microswimmers. Sci. Rep., $5,2015$.

41. N. Bertin, T. Spelman, O. Stephan, L. Gredy, M. Bouriau, E. Lauga, and P. Marmottant. Bubble-based acoustic micro-swimmers. Preprint.

42. B.K. Batchelor. An Introduction to Fluid Mechanics. Cambridge University Press, Cambridge, 1967.

43. Hideki Ishida and Yoshinobu Shigenaka. Cell model contraction in the ciliate Spirostomum. Cell Motil. Cytoskel., 9:278-282, 1988.

44. J.A. Gaunt. The Triplets of Helium. Phil. Trans. Roy. Soc. A, 228, 1929.

45. Yu-Lin Xu. Fast evaluation of the gaunt coefficients. Math. Comp., 65:1601-1612, October 1996.

46. E. Grosswald. Bessel Polynomials. Springer-Verlag Berlin Heidelberg, 1978.

47. M. S. Longuet-Higgins. Mass Transport in Water Waves. Phil. Trans. Roy. Soc. A, 245:535-581, 1953

48. V. Magar and T. J. Pedley. Average nutrient uptake by a self-propelled unsteady squirmer. J. Fluid Mech., 539:93-112, 2005.

49. T. Ishikawa, M. P. Simmonds, and T. J. Pedley. Hydrodynamic interaction of two swimming model micro-organisms. J. Fluid Mech., 568:119-160, 2006.

50. S. Michelin and E. Lauga. Efficiency optimization and symmetry-breaking in a model of ciliary locomotion. Phys. Fluids, 22, 2010.

51. S. R. Keller and T. Y. Wu. A porous prolate-spheroidal model for ciliated microorganisms. J. Fluid Mech., 80:259-278, 1977.

52. I.S.Aranson. Active colloids. Physics-Uspekhi, 56(1):79, 2013. 